University of Nebraska - Lincoln

DigitalCommons@University of Nebraska - Lincoln

Faculty Publications, Department of Mathematics

Mathematics, Department of

2012

\title{
R0 Analysis of a Spatiotemporal Model for a Stream Population
}

H. W. Mckenzie

Y. Jin

J. Jacobsen

M. A. Lewis

Follow this and additional works at: https://digitalcommons.unl.edu/mathfacpub

Part of the Applied Mathematics Commons, and the Mathematics Commons

This Article is brought to you for free and open access by the Mathematics, Department of at

DigitalCommons@University of Nebraska - Lincoln. It has been accepted for inclusion in Faculty Publications, Department of Mathematics by an authorized administrator of DigitalCommons@University of Nebraska - Lincoln. 


\title{
$R_{0}$ Analysis of a Spatiotemporal Model for a Stream Population*
}

\author{
H. W. Mckenzie ${ }^{\dagger}$, Y. $\operatorname{Jin}^{\dagger}$, J. Jacobsen ${ }^{\ddagger}$, and M. A. Lewis ${ }^{\dagger}$
}

\begin{abstract}
Water resources worldwide require management to meet industrial, agricultural, and urban consumption needs. Management actions change the natural flow regime, which impacts the river ecosystem. Water managers are tasked with meeting water needs while mitigating ecosystem impacts. We develop process-oriented advection-diffusion-reaction equations that couple hydraulic flow to population growth, and we analyze them to assess the effect of water flow on population persistence. We present a new mathematical framework, based on the net reproductive rate $R_{0}$ for advection-diffusion-reaction equations and on related measures. We apply the measures to population persistence in rivers under various flow regimes. This work lays the groundwork for connecting $R_{0}$ to more complex models of spatially structured and interacting populations, as well as more detailed habitat and hydrological data.
\end{abstract}

Key words. net reproductive rate, next generation operator, drift paradox, instream flow needs, spectral radius, positive operator

AMS subject classifications. $35 \mathrm{~K} 10,47 \mathrm{~A} 75,92 \mathrm{~B} 05$

DOI. $10.1137 / 100802189$

1. Introduction. Although industry, agriculture, and urban consumers place demands on water resources, society also values the conservation of stream ecosystems. Therefore water managers must design and implement ecologically sustainable water management programs. To do this they require estimates of the flows needed to maintain ecosystem integrity at a particular level, which are referred to as instream flow needs (IFNs). The unidirectional water flow in streams creates highly spatially and temporally variable environments and drives abiotic components of the ecosystem, including oxygen concentration, temperature distribution, and the cycling of organic matter and nutrients. Consequently, the alteration of hydrodynamic regimes associated with water management has direct effects on stream ecosystem dynamics [23].

Determining IFNs is challenging due to the complex and dynamic nature of the interaction between the stream environment and the biological community. Habitat simulation methods are the most widely used approach in North America. These employ habitat models and then correlate habitat conditions with the presence or absence of given species [29]. However, [2] argued for an alternative approach: process-oriented models that couple the population

\footnotetext{
* Received by the editors July 15, 2010; accepted for publication (in revised form) by J. Keener November 28, 2011; published electronically April 12, 2012.

http://www.siam.org/journals/siads/11-2/80218.html

${ }^{\dagger}$ Centre for Mathematical Biology, Department of Mathematical and Statistical Sciences, University of Alberta, Edmonton T6G2G1, Canada (hannah.mckenzie@gov.ab.ca, yujin@math.ualberta.ca, mlewis@math.ualberta.ca).

${ }^{\ddagger}$ Department of Mathematics, Harvey Mudd College, Claremont, CA 91711 (jacobsen@math.hmc.edu).

${ }^{\S}$ Department of Biological Sciences, University of Alberta, Edmonton T6G2G1, Canada.
} 
dynamics of river communities to the hydraulic dynamics of river flow. These models can provide estimates of IFNs based on the underlying ecological dynamics, rather than relying upon statistical correlations between habitat variables and the presence of species. We employ this alternative approach in what follows.

The class of process-oriented models for ecological dynamics of river populations that we consider stems from an interest in the "drift paradox." The drift paradox was first proposed by [21] following the observation that although individual organisms in streams are subject to downstream drift, the average location of the population is not observed to move downstream over time. Although no longer considered to be a true paradox [15], the search for potential explanations for the drift paradox motivated the development of a series of process-oriented models for populations in streams based on advection-diffusion-reaction equations $[26,22$, 19]. Similar models describe the interaction between flow and other ecological processes, for example, competition between species [20]. To date these models have been analyzed through the application of mathematical ideas such as traveling wave analysis and the critical domain size problem.

A paradox similar to the drift paradox exists in marine systems [24]. In this case, sedentary marine species release their planktonic larval stages into the water column, where they are dispersed by the currents before settling. Although there is a mean current, which is analogous to the downstream current in streams, populations are observed to maintain their position along the shoreline and even spread upstream, as observed when species expand their range or invade new areas. Several authors [3] studied this question using integro-difference equations to identify the conditions which allow marine species to spread against the mean current. They found that in addition to oceanographic parameters, retention depended on the total number of successful offspring produced over the lifetime of an individual in the absence of density dependent factors. They referred to this parameter as the "intrinsic capacity for increase over a lifetime" and emphasized its importance over the intrinsic growth rate as a measure of persistence for iteroparous species.

In spatially variable environments, such as those found in rivers, the intrinsic capacity for increase over a lifetime can depend upon the spatial location where an individual finds itself. Recently, [16] proposed three relevant measures of population persistence that relate to lifetime reproductive output in a spatially variable environment. The three measures are connected through the next generation operator, which maps the population forward in time from one generation to the next. We adapt these measures to provide a new way of analyzing the series of advection-diffusion-reaction models for populations in streams.

The first measure of persistence describes the fundamental niche of the species and we denote it $R_{\text {loc }}(x) .{ }^{1}$ It represents the local persistence and is the product of reproduction and survival at the location $x$. By definition it excludes dispersal. It is useful to think of $R_{\mathrm{loc}}(x)$ as answering the following question: if an individual is placed at location $x$ in the absence of dispersal, what will be its lifetime reproduction at that location? In the absence of dispersal, a population will persist at the location if $R_{\mathrm{loc}}(x)>1$ but will not persist if $R_{\mathrm{loc}}(x)<1$.

The second measure of persistence describes the realized niche and is denoted $R_{\delta}(x)$. This is the lifetime reproductive output of an individual initially introduced at $x$ undergoing

${ }^{1} R_{\mathrm{loc}}(x), R_{\delta}(x)$, and $R_{0}$ were termed $\hat{R}_{0}(x), R_{0}(x)$, and $\bar{R}_{0}$, respectively, by [16]. 
survival, reproduction, and dispersal. It is useful to think of $R_{\delta}(x)$ as answering the following question: if an individual is introduced at location $x$ and undergoes continuous dispersal and reproduction, what will be the lifetime reproductive output of that individual? Using $R_{\delta}(x)$ we can map the source and sink regions in the habitat. Although $R_{\delta}(x)$ incorporates the effect of abiotic factors, such as stream flow, on habitat suitability, it does not inform on the global persistence or extinction of a population. For this we turn to the final measure of persistence, the net reproductive rate, $R_{0}$.

The net reproductive rate $R_{0}$ has a long history as a measure of persistence in ecological modeling. In epidemiology, an analogous $R_{0}$ is defined and is called the basic reproduction number, representing the expected number of secondary infections caused by a single infected individual introduced into a susceptible population, and accordingly it can be used to predict persistence or eradication of a disease. In our work, $R_{0}$ is defined as the number of offspring produced by a single individual over its lifetime, assuming that the individual is subject to a particular spatial configuration in the river. As we will show, this spatial configuration is associated with the dominant eigenfunction of a next generation operator and as such can be interpreted as the spatial configuration associated with the maximum long-term intergenerational growth of the population. The analysis of $R_{0}$ aims to result in a simple and intuitive answer to the question of population persistence [8]. Intuitively, if $R_{0}>1$ the population will grow, but if $R_{0}<1$ the population will become extinct. The existence of this threshold makes $R_{0}$ a powerful tool for studying population persistence. As alluded to above, $R_{0}$ can be defined mathematically as the spectral radius of the next generation operator $[9,12]$. Note that $R_{0}$ is a dimensionless quantity and as such is not technically a rate. However, we continue to use this terminology to be consistent with the extensive biological literature in the area (see e.g., [5]).

Theory exists for proving the role of $R_{0}$ as a threshold parameter for populations described by matrix models [5, 7], ordinary differential equation models [32], and integro-differential equation models [16]. Recently such theory has also been established for reaction-diffusion equations in epidemiology [18, 33, 31]. As spatial reaction-advection-diffusion equations fall under the broader category of infinite-dimensional dynamical systems, and recent work by [30] on $R_{0}$ in infinite-dimensional dynamical systems can be applied, in the current work we develop the theory for $R_{0}$, as well as $R_{\mathrm{loc}}(x)$ and $R_{\delta}(x)$, in spatially heterogeneous reactionadvection-diffusion equation that describes stream populations. We do this by adapting and applying the abstract theory of $R_{0}$ for an infinite-dimensional population structure given in [30]. This is the first work applying the basic reproduction number to parabolic spatial PDEs in nondisease context. The mathematical methods are then applied to our scientific goal - to provide a new way of analyzing IFN for populations in rivers.

The paper is divided into two sections. In the theory section we introduce a general model for a population in a stream and formulate the associated next generation operator. Using this next generation operator we derive three measures of persistence proposed by [16] for our model. In particular, using the results of [30] we show that in this case $R_{0}$ can be used to determine the stability of the homogeneous trivial steady state of the population model and can therefore be used as a measure of global population persistence. In the applications section we analyze two models for populations in steams using the measures of persistence derived in the theory section.

Copyright (c) by SIAM. Unauthorized reproduction of this article is prohibited. 


\section{Theory.}

2.1. A general model for a population in a stream. We study the following general model for a single population undergoing continuous growth and dispersal in a stream of length $L$ :

$$
\begin{cases}n_{t}=g(x, n) n-\frac{Q}{A(x)} n_{x}+\frac{1}{A(x)}\left(D(x) A(x) n_{x}\right)_{x}, & x \in(0, L), t>0, \\ \alpha_{1} n(0, t)-\beta_{1} n_{x}(0, t)=0, & t>0, \\ \alpha_{2} n(L, t)+\beta_{2} n_{x}(L, t)=0, & t>0, \\ n(x, 0)=n_{0}(x), & x \in(0, L),\end{cases}
$$

where $n(x, t)$ is the population density at location $x$ and time $t, g(x, n)$ is the per capita growth rate function, $A, D \in C^{2}([0, L],(0, \infty))$ are the cross-sectional area of the stream and the spatially variable diffusion coefficient, respectively, $Q>0$ is the constant stream discharge, $\alpha_{i}$ and $\beta_{i}$ are nonnegative constants $(i=1,2)$, and $n_{0}$ is the initial distribution of the population. We define the strongly elliptic linear operator

$$
\mathcal{L}:=-\frac{Q}{A(x)} \frac{\partial}{\partial x}+\frac{1}{A(x)} \frac{\partial}{\partial x}\left(D(x) A(x) \frac{\partial}{\partial x}\right)
$$

which represents both the directed dispersal due to downstream flow and the random dispersal due to turbulence and intrinsic movement of individuals, respectively. (See [19] for the derivation of (2.1) from a three-dimensional conservation law for movement of individuals in streams.) The governing equation in (2.1) can then be written as

$$
n_{t}=g(x, n) n+\mathcal{L} n .
$$

Applying the product rule in (2.2) one sees that the operator can be expressed in the alternate form

$$
\widetilde{\mathcal{L}}:=-a(x) \frac{\partial}{\partial x}+D(x) \frac{\partial^{2}}{\partial x^{2}}
$$

where $a(x)=\frac{Q}{A(x)}-D^{\prime}(x)-\frac{D(x) A_{x}(x)}{A(x)}$. Although the operator $\mathcal{L}$ is specific to dispersal in streams, all the results in this paper hold if $\mathcal{L}$ is replaced by a strongly elliptic operator in the form (2.4).

We assume that the growth rate function $g(x, n)$ satisfies the following:

$\mathrm{H}(\mathrm{i}) \mathrm{g}:[0, L] \times(0, \infty) \rightarrow \mathbb{R}$ is continuous.

$\mathrm{H}$ (ii) $g(x, n)$ is monotonically decreasing in $n$ and for each $x$ there exists a unique value $n(x):=K(x)>0$ such that $g(x, K(x))=0$.

$\mathrm{H}\left(\right.$ iii) and $g(x, 0):=\lim _{n \rightarrow 0^{+}} g(x, n)<\infty$.

Notice from property $\mathrm{H}(\mathrm{ii})$ that we are not allowing for an Allee effect and each location has an associated carrying capacity $K(x)>0$. The limiting value in property $\mathrm{H}$ (iii) represents the intrinsic growth rate at location $x$. We assume $g(x, 0)=f(x)-v(x)$, where $f(x)>0$ and $v(x)>0$ are spatially varying intrinsic birth rate and mortality rate, respectively.

The boundary conditions we consider are either Dirichlet $\left(\alpha_{i}=1, \beta_{i}=0\right)$, Neumann $\left(\alpha_{i}=0, \beta_{i}=1\right)$, or Robin $\left(\alpha_{i} \geq 0, \beta_{i} \geq 0, \alpha_{i}+\beta_{i} \neq 0\right)$ conditions. In particular, we allow 
for two types of boundary conditions relevant to streams, which we refer to as hostile and Danckwerts boundary conditions. Hostile conditions represent zero-flux at the stream source (individuals cannot enter or leave the domain at the source) and zero-density at the stream outflow (e.g., the stream discharges all individuals into a region such as a lake or a waterfall, from which they cannot return) [26]:

$$
Q n(0, t)-D(0) A(0) n_{x}(0, t)=0 \text { and } n(L, t)=0 .
$$

Danckwerts conditions also assume zero-flux at the stream source but use a free-flow or insulated condition at the stream outflow:

$$
Q n(0, t)-D(0) A(0) n_{x}(0, t)=0 \text { and } n_{x}(L, t)=0 .
$$

For a derivation and discussion of these boundary conditions from a random-walk perspective, see [19].

Our approach to population persistence and extinction is based on the following mathematical setting. Let $X=C[0, L]$ denote the Banach space of continuous functions on the interval $[0, L]$ with the supremum norm $\|u\|_{\infty}=\max _{x \in[0, L]}|u(x)|$ for $u \in X$. The nonnegative functions $X_{+}=\{f \in X: f(x) \geq 0$ for all $x \in[0, L]\}$ then form a (solid) cone in $X$ (the cone of nonegative functions). The well-posedness and comparison principle for (2.1) are standard results on $X$ (see, e.g., [4]).

Note that $n^{*} \equiv 0$ is the trivial homogeneous steady state solution of (2.1) and the associated linearized system of (2.1) at $n^{*}$ is

$$
\begin{cases}n_{t}=g(x, 0) n+\mathcal{L} n, & x \in(0, L), t>0, \\ \alpha_{1} n(0, t)-\beta_{1} n_{x}(0, t)=0, & t>0, \\ \alpha_{2} n(L, t)+\beta_{2} n_{x}(L, t)=0, & t>0, \\ n(x, 0)=n_{0}(x), & x \in(0, L) .\end{cases}
$$

We consider the eigenvalue problem

$$
\left\{\begin{array}{l}
g(x, 0) n+\mathcal{L} n=\mu n, \quad x \in(0, L), \\
\alpha_{1} n(0)-\beta_{1} n_{x}(0)=0, \\
\alpha_{2} n(L)+\beta_{2} n_{x}(L)=0
\end{array}\right.
$$

and define $\mu_{1}$ to be the principal eigenvalue of (2.8). (The existence of $\mu_{1}$ follows from, e.g., [4, Corollary 2.13].) By similar arguments as in the proofs of Propositions 3.1 and 3.2 in [4], we obtain the following proposition. Similar results can be found in Chapter 7 of [25] (e.g., Theorem 6.2, which applies to more general equations as per the remark on p. 121).

Proposition 2.1. For (2.1) with g satisfying $\mathrm{H}(\mathrm{i})-\mathrm{H}(\mathrm{iii})$, the following statements are valid:

(i) If $\mu_{1}<0$, then the trivial steady state $n^{*}=0$ of (2.1) is locally asymptotically stable.

(ii) If $\mu_{1}>0$, then $n^{*}=0$ is unstable. Moreover, (2.1) admits a minimal positive equilibrium $\hat{n}(x)$ and all solutions of (2.1) which are initially positive on an open subset of $[0, L]$ are eventually bounded below by solutions (with different initial data) which increase toward $\hat{n}(x)$ as $t \rightarrow \infty$.

Copyright (c) by SIAM. Unauthorized reproduction of this article is prohibited. 
Note that in (ii), by "minimal" we mean that if $\tilde{n}$ is another positive equilibrium, then $\hat{n}(x) \leq \tilde{n}(x)$ for all $x \in[0, L]$. In particular, when $\mu_{1}>0$, we are not concerned with how many positive equilibria the system has, although we expect that there is only one. A minimal positive equilibrium is sufficient for our analysis.

The following corollary of Proposition 2.1 motivates our notion of invasion and persistence (for the proof see Appendix A.1).

Proposition 2.2. Let $\mu_{1}$ be the principal eigenvalue of (2.8). If $\mu_{1}>0$, then (2.1) is uniformly persistent in the sense that there exists $\delta>0$ such that for any solution $n(x, t)$ of (2.1) with $n(x, 0)=n_{0} \in X_{+} \backslash\{0\}$ we have

$$
\liminf _{t \rightarrow \infty} \min _{x \in[0, L]} n(x, t) \geq \delta
$$

when the boundary conditions in (2.1) are Neumann or Robin conditions and

$$
\liminf _{t \rightarrow \infty} \max _{x \in[0, L]} n(x, t) \geq \delta
$$

when at least one of the boundary conditions in (2.1) are Dirichlet conditions.

Following Proposition 2.2, in this paper we say that a population described by (2.1) will invade and persist in the stream if $\mu_{1}>0$ but will be washed out if $\mu_{1}<0$. Therefore, to determine if a population will invade and persist in a stream, it suffices to study the sign of $\mu_{1}$. Note that in this paper, we do not address the case where $\mu_{1}=0$. When the parameters $f, v, A, D, Q$ are constants, the system (2.7) reduces to the model of [26], which is well understood through traveling wave and critical domain size analysis (reviewed in [17] and further discussed in Example $\mathrm{A}$ in section 3). In the following sections, we develop the theory necessary to study the behavior of the full model using the different spatial measures of persistence discussed in the introduction.

2.2. Definition of the next generation operator. In this section we define the next generation operator associated with the spatial model (2.7). The operator maps an initial population distribution to its "next generation" distribution. By the study of the properties of the next generation operator, we expect to see whether the population can persist in a stream.

Suppose a population of species is introduced into the stream environment $[0, L]$ with distribution $\psi_{0} \in X$ and then these individuals experience dispersal and reproduction until they die. If $\psi(x, t)$ denotes the distribution of these initial individuals at time $t$, then their density over time is governed by the model

$$
\begin{cases}\psi_{t}=-v(x) \psi+\mathcal{L} \psi, & x \in(0, L), t>0, \\ \psi(x, 0)=\psi_{0}(x), & x \in(0, L)\end{cases}
$$

where $v(x)>0$ is their mortality rate. The function $f(x) \psi(x, t)$ is then the rate of population production by the initial individuals at location $x$ at time $t$, and therefore the total reproduction of the initial individuals during their lifetime is given by the function $\int_{0}^{\infty} f(x) \psi(x, t) d t$, which we call the next generation distribution. This leads us to the following definition.

Copyright (c) by SIAM. Unauthorized reproduction of this article is prohibited. 
Definition 2.3. The next generation operator $\Gamma: X \rightarrow X$ associated with (2.7) is defined by

$$
\Gamma \psi_{0}(x)=\int_{0}^{\infty} f(x) \psi(x, t) d t=f(x) \int_{0}^{\infty} \psi(x, t) d t
$$

where $\psi(x, t)$, the distribution of the initial individuals at time $t$, is the classical solution of (2.11) subject to the same boundary conditions as (2.7).

The following proposition shows that $\Gamma$ is a well-defined operator on $X$. (See Appendix A.2 for the proof.)

Proposition 2.4. The operator $\Gamma$ is defined for all $\psi_{0} \in X$ and $\Gamma \psi_{0} \in X$.

Alternatively, it is possible to define the next generation operator $\Gamma$ by way of the solution of an associated boundary value problem, as described in the following proposition.

Proposition 2.5. The next generation operator defined by (2.12) can alternatively be defined by

$$
\Gamma \psi_{0}(x)=f(x) \int_{0}^{L} k(x, y) \psi_{0}(y) d y,
$$

where $k(x, y)$ is the solution of the ordinary boundary value problem

$$
\left\{\begin{array}{l}
\mathcal{L} k(x, y)-v(x) k(x, y)=-\delta(x-y), \quad x \in(0, L) \\
\alpha_{1} k(0, y)-\beta_{1} k^{\prime}(0, y)=0 \\
\alpha_{2} k(L, y)+\beta_{2} k^{\prime}(L, y)=0
\end{array}\right.
$$

for a fixed value of $y$. (Here' denotes differentiation with respect to x.)

The function $k(x, y)$ can be considered as the lifetime density of space use of an individual initially introduced at $y$. A benefit of this alternative formulation of the next generation operator is that it reduces the problem of solving a partial differential equation to that of solving a boundary value problem with an ordinary differential equation. This simplifies both analytical and numerical computation of $\Gamma \psi_{0}(x)$. The proof of Proposition 2.5 relies on first proving the special case where $f$ is constant. In this case, judicious differentiation and integration of (2.11) yields a boundary value problem for $\Gamma \psi_{0}(x)$. The solution to this problem can be expressed using Green's function, which yields the definition (2.13). The proof for general $f(x)$ follows directly from the case where $f$ is constant. See Appendix A.3 for details.

2.3. Three measures of population persistence. In this section we show how three measures of persistence for populations described by the model (2.7) can be understood in terms of the next generation operator $\Gamma$ defined by (2.12). We apply these ideas to two specific examples in section 3 .

Measure 1: $\boldsymbol{R}_{\text {loc }}(x)$. The first measure of persistence, denoted $R_{\text {loc }}(x)$, determines the fundamental niche of the species. Recall that the fundamental niche of an organism in its ecosystem is the full range of environmental conditions and resources (biological and physical) that the organism can possibly occupy and use, especially when limiting factors are absent

Copyright (C) by SIAM. Unauthorized reproduction of this article is prohibited. 
in its habitat. By definition $R_{\mathrm{loc}}(x)$ excludes dispersal, so we assume that the individual only experiences birth and death after being introduced into the stream and exclude dispersal processes during its lifetime. Then (2.7) reduces to

$$
\begin{cases}n_{t}=g(x, 0) n, & x \in(0, L), t>0, \\ n(x, 0)=n_{0}(x), & x \in[0, L] .\end{cases}
$$

Therefore, the number of offspring produced by an individual introduced at $x \in[0, L]$ over its lifetime is

$$
R_{\mathrm{loc}}(x):=\left(\Gamma \psi_{0}\right)(x)=f(x) \int_{0}^{\infty} \psi(x, t) d t
$$

where $\psi(x, t)$ is the solution to the initial-value problem

$$
\left\{\begin{array}{l}
\psi_{t}(x, t)=-v(x) \psi(x, t), \quad t>0, \\
\psi(x, 0)=1
\end{array}\right.
$$

where $x$ is considered as a parameter. From $(2.17)$ we see $\psi(x, t)=e^{-v(x) t}$ and therefore

$$
R_{\mathrm{loc}}(x)=f(x) \int_{0}^{\infty} e^{-v(x) t} d t=\frac{f(x)}{v(x)} .
$$

It follows from the meaning of the next generation operator that if $R_{\text {loc }}(x)>1$, an individual introduced at $x$ will produce more than one offspring at $x$ in the next generation and the population at $x$ will increase over the generations. Therefore, locations with $R_{\text {loc }}(x)>1$ correspond to the fundamental niche of the species. Note that $R_{\mathrm{loc}}(x)>1$ is equivalent to $g(x, 0)=f(x)-v(x)>0$, i.e., the population growth rate at $x$ is positive, and that $R_{\text {loc }}(x)<1$ is equivalent to $g(x, 0)<0$, so we can use the sign of $g$ to determine if $R_{\mathrm{loc}}(x)>1$.

Measure 2: $\boldsymbol{R}_{\delta}(x)$. For the second measure of persistence, denoted $R_{\delta}(x)$, we return to the full spatial model (2.7), now in the context of introducing a single individual at location $x$. The function $R_{\delta}(x)$ describes the number of offspring produced by an individual introduced at $x$ and undergoing birth, death, and dispersal dynamics. It determines the source-sink distribution in the stream. In this case the next generation distribution from a single individual introduced at $x$ is defined by

$$
\Gamma \psi_{0}(z)=f(z) \int_{0}^{\infty} \psi(z, t) d t, \quad z \in[0, L]
$$

where $\psi(z, t)$ is the solution of (2.11) with $\psi_{0}(z)=\delta(z-x)$ and $\delta(\cdot)$ is the Dirac delta distribution. We then define $R_{\delta}(x)$ by

$$
R_{\delta}(x):=\int_{0}^{L} \Gamma \psi_{0}(z) d z
$$

Copyright (c) by SIAM. Unauthorized reproduction of this article is prohibited. 
It follows from the alternative definition for the next generation operator in Proposition 2.5 that we can simplify $R_{\delta}(x)$ as

$$
R_{\delta}(x)=\int_{0}^{L} \Gamma \psi_{0}(z) d z=\int_{0}^{L} f(z) \int_{0}^{L} k(z, y) \delta(y-x) d y d z=\int_{0}^{L} f(z) k(z, x) d z,
$$

where $k$ is defined by (2.14). If $R_{\delta}(x)>1$, then an individual introduced at $x$ will produce more than one offspring somewhere in the stream in the next generation and therefore the location $x$ acts as a source. Similarly, if $R_{\delta}(x)<1$, then $x$ serves as a sink. Thus, $R_{\delta}(x)$ is a measure of the source-sink dynamics in the stream.

Measure 3: $\boldsymbol{R}_{0}$. For the final measure of persistence, denoted $R_{0}$, we consider the full spatial model (2.7) now in the context of arbitrary initial distributions. For any distribution $\psi_{0}(x)$ we can consider the associated next generation distribution

$$
\Gamma \psi_{0}(x)=\int_{0}^{\infty} f(x) \psi(x, t) d x,
$$

where $\psi(x, t)$ solves (2.11). Define

$$
R_{0}:=r(\Gamma)
$$

where $r(\Gamma)$ is the spectral radius (see, e.g., [9] and section 2.4) of the linear operator $\Gamma$ on $X$. We call $R_{0}$ the net reproductive rate. Although $R_{0}$ cannot be thought of conceptually in terms of defining the fundamental niche or source-sink regions, it does provides a global measure of population persistence for the spatial model (over all initial conditions) and has implications for the long-term behavior of the population. In fact, $r(\Gamma)$ represents the number of offspring an individual may produce in its next generation. In the next section we outline the key properties of $R_{0}$ before considering applications to streams in section 3 .

2.4. Persistence and $R_{0}$ : Spectral properties of the next generation operator. In this section we outline the key properties of the net reproductive rate $R_{0}$ defined by $(2.22)$ and relate it to persistence of populations in streams by demonstrating its relation with the stability of the trivial homogeneous steady state solution $n^{*} \equiv 0$ of the linearized population model (2.7). We also summarize a useful projection method for numerically approximating $R_{0}$ due to [6].

To motivate the mathematical ideas in this section we briefly review the situation for the finite-dimensional setting of structured population models. Consider the model

$$
x_{t+1}=A x_{t}
$$

for a structured population $x=\left(x_{1}, x_{2}, \ldots, x_{n}\right)$ governed by the transition matrix $A$. If $A$ is a nonnegative, primitive matrix, then the Perron-Frobenius theorem implies the magnitude of the largest eigenvalue of $A$ is in fact an eigenvalue, known as the dominant eigenvalue $\lambda_{1}$. If $\lambda_{1}>1$, the zero solution of (2.23) is unstable and the population will grow exponentially (persistence). On the other hand, if $\lambda_{1}<1$, the zero solution is stable and the population will

Copyright (c) by SIAM. Unauthorized reproduction of this article is prohibited. 
die out (extinction). If we write $A$ as the sum $A=F+T$, where $F$ represents fecundity and $T$ transitions between classes, then the next generation operator associated with (2.23) is the matrix

$$
R=F(I-T)^{-1}
$$

which models the net population dynamics due to a single cohort $x$ in terms of their offspring $F x$, their transition's offspring $F T x$, the subsequent offspring $F T^{2} x$, and so on. If the spectral radius of $\mathrm{T}$ satisfies $r(T)<1$, then the net contribution due to $x$ will be

$$
F x+F T x+F T^{2} x+\cdots=F\left(I+T+T^{2}+T^{3}+\cdots\right) x=F(I-T)^{-1} x=R x .
$$

The finite-dimensional net reproductive number $R_{0}$ is then defined to be the spectral radius (i.e., magnitude of largest eigenvalue) of the matrix $R$. It can be shown that the dominant eigenvalue of (2.23) satisfies $\lambda_{1}>1$ if and only if $R_{0}>1$ [7]. In [7], the authors also provide examples where the $R_{0}$ framework provides additional insight into the population model due to the availability of explicit algebraic formulas for $R_{0}$ that are not available for the eigenvalue $\lambda_{1}$.

Our approach to studying persistence in streams parallels that of finite-dimensional population models such as (2.23), although now in the infinite-dimensional setting. The operator $\Gamma$ defined by (2.12) is the analogue of (2.24).

We briefly review the appropriate definitions. If $S$ is a Banach space and $T: S \rightarrow S$ is a bounded linear operator, then the spectrum of $T$ is the set $\sigma(T)=\{\lambda \in \mathbb{C} \mid \lambda I-T$ is not invertible $\}$. The spectral radius of $T$ is defined to be $r(T)=\sup \{|\lambda|: \lambda \in \sigma(T)\}$ and the spectral bound of $T$ is defined by $s(T)=\sup \{\operatorname{Re}(\lambda): \lambda \in \sigma(T)\}$. The complement of $\sigma(T)$ is known as the resolvent set of $T$ and denoted $\rho(T)=\mathbb{C} \backslash \sigma(T)$ [14]. Our methods rely on the Krein-Rutman theorem for compact operators, which is an infinite-dimensional analogue of the Perron-Frobenius theorem. A bounded linear operator $T: S \rightarrow S$ is compact if for each bounded sequence $\left\{u_{k}\right\}_{k=1}^{\infty} \subset S$ there exists a subsequence $\left\{u_{k_{j}}\right\}_{j=1}^{\infty}$ such that $\left\{T u_{k_{j}}\right\}_{j=1}^{\infty}$ converges in $S$.

Proposition 2.6. The next generation operator defined by (2.12) is a bounded, compact linear operator on the space $X=C[0, L]$.

Proof. Clearly $\Gamma$ is a linear operator, and by Proposition 2.4 we know $\Gamma: X \rightarrow X$. Compactness follows from an application of the Arzelà-Ascoli theorem. We provide the full details in Appendix A.4.

The operator analogue for positivity of matrices is defined in terms of cones. A cone $K \subset S$ is a closed, convex set such that $\alpha K \subset K$ for all $\alpha \geq 0$ and $K \cap(-K)=\{0\}$. A cone $K$ in the Banach space $S$ induces a partial order $\leq$ by the rule $u \leq v$ if $v-u \in K$. If $K$ has a nonempty interior it is called a solid cone. The operator $T$ is strongly positive if for all $u \in K \backslash\{0\}$ it follows $T u \in \operatorname{Int}(K)$. The following theorem is an infinite-dimensional analogue of the Perron-Frobenius theorem.

Theorem 2.7 (Krein-Rutman theorem, [10, Theorems 1.1 and 1.2]). Let $S$ be a Banach space, $K \subset S$ a solid cone, and $T: S \rightarrow S$ a strongly positive, compact linear operator. Then

(i) $r(T)>0$ and $r(T)$ is a simple eigenvalue with a positive eigenvector $\phi \in \operatorname{Int}(K)$, and there is no other eigenvalue with a positive eigenvector;

(ii) $r(T)$ is a dominant eigenvalue, i.e., $|\lambda|<r(T)$ for all eigenvalues $\lambda \neq r(T)$.

Copyright (c) by SIAM. Unauthorized reproduction of this article is prohibited. 
Applying the Krein-Rutman theorem to the next generation operator $\Gamma$ on $X$ yields the following result for $R_{0}$.

Proposition 2.8. The net reproductive rate $R_{0}$ is the dominant eigenvalue of the next generation operator $\Gamma$ defined by (2.12). Furthermore, $R_{0}$ is a simple eigenvalue and is the only eigenvalue with a positive eigenfunction (positive on $(0, L)$ for Dirichlet boundary conditions and positive on $[0, L]$ otherwise).

Proof. The proof depends on the boundary conditions under consideration. When the boundary conditions are Neumann or Robin boundary conditions, one can directly apply the Krein-Rutman theorem to the operator $\Gamma$ on $X$; when one or more boundary conditions are Dirichlet boundary conditions, then the result follows by considering $\Gamma$ on a closed subspace of continuously differentiable functions which vanish at the boundary. The full proof is given in Appendix A.5.

The relation between $R_{0}$ and the stability of the trivial homogeneous steady state solution of (2.7) follows from results concerning resolvent-positive operators in [30]. The operator $T$ is resolvent-positive if the resolvent set $\rho(T)$ contains a ray $(\omega, \infty)$ and $(\lambda I-T)^{-1}$ is a positive operator for all $\lambda>\omega$ [30]. The following result of [30] is the key to connecting the next generation operator to stability of the trivial solution.

Theorem 2.9 (see [30, Theorem 3.5]). Let $B$ be a resolvent-positive operator in the ordered Banach space $S$ with $s(B)<0$. If $C$ is a positive linear operator such that $A=B+C$ is also resolvent-positive, then $s(A)$ has the same sign as $r\left(-C B^{-1}\right)-1$.

Theorem 2.9 generalizes results in [32] for ordinary differential equations to the infinitedimensional setting. In our context of a stream population we will show that there exist operators $C$ and $B$ such that $A=B+C$ is the operator $A n=g(x, 0) n+\mathcal{L} n$ (the right-hand side of the differential equation in (2.7)) and the next generation operator $\Gamma$ defined by $(2.12)$ satisfies

$$
\Gamma=-C B^{-1}
$$

Thus the net reproductive rate $R_{0}$ satisties

$$
R_{0}=r\left(-C B^{-1}\right)
$$

Assuming $B$ and $C$ satisfy the hypothesis of Theorem 2.9, it follows from Theorem 2.9 that $R_{0}$ determines the stability of the trivial steady state solution of the associated population model (2.7).

Proposition 2.10. Consider the set $Z$ of nonnegative $C^{2}$ functions on $[0, L]$ that satisfy the general boundary conditions given in (2.1), i.e.,

$$
\begin{aligned}
& Z=\left\{w: w \in C^{2}([0, L], \mathbb{R}), w \geq 0 \text { for all } x \in[0, L], \alpha_{1} w(0)-\beta_{1} w^{\prime}(0)=0,\right. \\
& \left.\quad \text { and } \alpha_{2} w(L)+\beta_{2} w^{\prime}(L)=0\right\} .
\end{aligned}
$$

Then the next generation operator defined in (2.12) satisfies $\Gamma=-C B^{-1}$, where $B$ and $C$ are defined by

$$
\begin{aligned}
& B w(x)=\mathcal{L} w(x)-v(x) w(x), \\
& C w(x)=f(x) w(x)
\end{aligned}
$$

Copyright $\odot$ by SIAM. Unauthorized reproduction of this article is prohibited. 
for any $w \in Z$. Moreover, for

$$
A=B+C,
$$

the spectral bound $s(A)$ has the same sign as $R_{0}-1$, where $R_{0}=r\left(-C B^{-1}\right)=r(\Gamma)$.

We present the proof of this proposition in Appendix A.6. By applying Theorem 2.9 to (2.1) with operators $A, B$, and $C$ as defined in the above proposition, we obtain the following theorem.

Theorem 2.11. Let $\Gamma$ be the next generation operator defined by (2.12) and let $R_{0}=r(\Gamma)$ be the spectral radius of $\Gamma$. For the population model (2.1), the homogeneous trivial steady state solution $n^{*} \equiv 0$ is locally asymptotically stable when $R_{0}<1$ and unstable when $R_{0}>1$. Moreover, if $R_{0}>1$, then the population is uniformly persistent.

Proof. Note that the existence of the principal eigenvalue $\mu_{1} \in \mathbb{R}$ of $A$ follows from the proofs of Propositions 3.1 and 3.2 in [4], which implies the spectral bound $s(A)=\mu_{1}$. By Proposition 2.10, $s(A)$ has the same sign as $R_{0}-1$, so $s(A)<0$ if and only if $R_{0}<1$ and $s(A)>0$ if and only if $R_{0}>1$. Finally, Proposition 2.1 implies that when $R_{0}<1$, $n^{*}$ is locally asymptotically stable, and when $R_{0}>1, n^{*}$ is unstable and the population is uniformly persistent.

Theorem 2.11 is a special case of Theorems 3.16 and 3.17 in [30]. It relates $R_{0}$ to the long-term behavior of a population described by (2.7) and to the local behavior near the homogeneous trivial steady state of populations described by (2.1). Therefore, $R_{0}$ is a measure of population invasion and persistence in streams.

We conclude this section by presenting a method for approximating $R_{0}$. It follows from Proposition 2.8 that we can find $R_{0}$ by solving the eigenvalue problem

$$
\Gamma \phi=R_{0} \phi
$$

where $\phi(x)$ is a positive function associated with the dominant eigenvalue $R_{0}$ of the infinitedimensional operator $\Gamma$. In some cases it is possible to find an analytic expression for $R_{0}$ (see, e.g., Example A in section 3). For cases where analytic solutions are not possible, the projection method (see, e.g., [6, section 3.1.1]) provides a practical approach for approximating $R_{0}$. To apply this method, we first approximate the Banach space $X=C[0, L]$ by $X_{n}(n \in \mathbb{N})$, the set of piecewise linear functions on $\left\{0=x_{1}<x_{2}<\cdots<x_{n}=L\right\}$ (equally spaced). The space $X_{n}$ has basis $e_{i}(x)$, where $e_{i}(x)$ is the piecewise linear function with $e_{i}\left(x_{i}\right)=1$ and $e_{i}\left(x_{j}\right)=0$ for $j \neq i$. Then the eigenvalue/function pairs $(\lambda, \phi)$ that satisfy $\Gamma \phi=\lambda \phi$ are approximated by $\left(\lambda_{n}, \phi_{n}\right)$, which satisfy $\Gamma_{n} \phi_{n}=\lambda_{n} \phi_{n}$, where $\Gamma_{n}$ is the matrix with entries

$$
\left(\Gamma_{n}\right)_{i j}=\int_{x_{j-1}}^{x_{j+1}} f\left(x_{i}\right) k\left(x_{i}, s\right) e_{j}(s) d s \quad \text { for } i, j=1, \ldots, n,
$$

and $k$ is as in (2.14). Thus, $R_{0}$ can be approximated by the dominant eigenvalue of the $n \times n$ matrix $\Gamma_{n}$ for very large $n$.

3. Applications. In this section we consider two examples of the $R_{0}$ theory. In Example $\mathrm{A}$ we use $R_{0}$ to revisit the critical domain size problem for (2.7) under the assumption that $g, Q, A$ and $D$ are constants as first developed by [26]. In particular, we graphically demonstrate the

Copyright (c) by SIAM. Unauthorized reproduction of this article is prohibited. 
theoretical connection between the population dynamics and the next generation operator $\Gamma$. In Example B we consider all three measures of persistence formulated in section 2.3 to study a similar model, now with variable growth rate $f$, which expands upon work in [20] for a single species in a stream with variable growth rate.

Example A: Revisiting critical domain size for populations in streams. We consider the special case of (2.7) where $g, Q, A$, and $D$ are constants. Under this assumption, (2.7) with a hostile boundary condition reduces to a model for which the critical domain size problem was first studied in [26]:

$$
\begin{cases}n_{t}=(f-v) n-a n_{x}+D n_{x x}, & x \in(0, L), t>0, \\ a n(0, t)-D n_{x}(0, t)=0, n(L, t)=0, & t>0, \\ n(x, 0)=n_{0}(x), & x \in(0, L) .\end{cases}
$$

This model is well understood through critical domain size analysis, which refers to finding the minimum stream length required for a population to persist in the stream. In particular, the critical domain size for (3.1) is

$$
L_{\text {crit }}=\frac{\sqrt{D / r}}{\sqrt{1-a^{2} /(4 r D)}}\left(\arctan \left(-\frac{2 \sqrt{r D}}{v} \sqrt{1-a^{2} /(4 D r)}\right)+\pi\right) .
$$

The result in (3.2) was shown by [26] (online appendix) and was restated in [17]. (Note that the original expression for the critical domain size in [26] should be adjusted to (3.2).)

We now show that the critical domain size $L_{\text {crit }}$ can also be found by analyzing the next generation operator. From Theorem 2.11 the threshold for persistence of a population in a domain of length $L$ occurs when $R_{0}=1$. Recall that $R_{0}$ is the dominant eigenvalue of the next generation operator associated with (3.1); therefore we solve the eigenvalue problem

$$
\Gamma \phi(x)=\int_{0}^{\infty} f \psi(x, t) d t=\lambda \phi(x),
$$

where $\psi(x, t)$ solves

$$
\begin{cases}\psi_{t}=-v \psi-a \psi_{x}+D \psi_{x x}, & x \in(0, L), t>0, \\ a \psi(0, t)-D \psi_{x}(0, t)=0, \psi(L, t)=0, & t>0 \\ \psi(x, 0)=\phi(x), & x \in(0, L)\end{cases}
$$

to determine for what values of $L$ we have $R_{0}>1$. Differentiating $\Gamma \phi$ and using Proposition 2.5 it follows that $\Gamma \phi(x)$ solves

$$
\left\{\begin{array}{l}
-v(\Gamma \phi)(x)-a(\Gamma \phi)^{\prime}(x)+D(\Gamma \phi)^{\prime \prime}(x)=-f \phi(x), \quad x \in(0, L), \\
a(\Gamma \phi)(0)-D(\Gamma \phi)^{\prime}(0)=0,(\Gamma \phi)(L)=0,
\end{array}\right.
$$

Copyright $\odot$ by SIAM. Unauthorized reproduction of this article is prohibited. 
where the boundary conditions follow from (3.3) and the boundary conditions on $\psi$ in (3.4). Since $\Gamma \phi=\lambda \phi$ we obtain the following Sturm-Liouville equation for $\phi(x)$ :

$$
\left\{\begin{array}{l}
\phi^{\prime \prime}(x)-\frac{a}{D} \phi^{\prime}(x)+\frac{1}{D}\left(\frac{f}{\lambda}-v\right) \phi(x)=0, \quad x \in(0, L), \\
a \phi(0)-D \phi^{\prime}(0)=0, \phi(L)=0 .
\end{array}\right.
$$

The characteristic equation for (3.6) is $\eta^{2}-\frac{a}{D} \eta+\frac{1}{D}\left(\frac{f}{\lambda}-v\right)=0$ with discriminant $\Delta=$ $(a / D)^{2}-4(f / \lambda-v) / D$. It is straightforward to show that there is no positive solution of (3.6) when $\Delta \geq 0$ due to the boundary conditions. Thus positive solutions of (3.6) exist only when $\Delta<0$ or, equivalently, when

$$
a^{2}<4 D\left(\frac{f}{\lambda}-v\right)
$$

Recall from Theorem 2.11 that persistence in a domain of length $L$ occurs when $\lambda=R_{0} \geq 1$. If $\lambda \geq 1$, then $f-v \geq f / \lambda-v$, in which case (3.7) implies

$$
a<2 \sqrt{D(f-v)} \leq 2 \sqrt{D(f-v)} .
$$

Recall that

$$
c^{*}:=2 \sqrt{D(f-v)}
$$

is the spreading speed for a population in the absence of advection (see, e.g., [17]). Thus $a<c^{*}$ is a necessary condition for the population to persist, i.e., the advective speed of the river must be less than the spreading speed of the population in the absence of advection. If $a>c^{*}$, then either there is no positive solution of (3.6) or a positive solution exists but $\lambda<1$, which in either case implies that the zero solution is stable and the critical domain size does not exist.

If $a<c^{*}$, then solving (3.6) one finds that the eigenvalues $\lambda$ satisfy

$$
\tan (\rho(\lambda) L)=-\frac{2 D \rho(\lambda)}{a}
$$

where $\rho(\lambda)=\sqrt{(f / \lambda-v) / D-(a / 2 D)^{2}}$. Implicitly differentiating (3.9) with respect to $L$ one sees that $d \lambda / d L>0$. Thus if we substitute $\lambda=R_{0}=1$ into (3.9) we obtain the critical domain size $L_{\text {crit }}$ as the minimal positive solution of (3.9), which yields

$$
L_{\text {crit }}=\frac{1}{\sqrt{(f-v) / D-(a / 2 D)^{2}}}\left(\arctan \left(-\frac{2 D \sqrt{(f-v) / D-(a / 2 D)^{2}}}{a}\right)+\pi\right),
$$

which is equivalent to $L_{\text {crit }}$ in (3.2) with $r=f-v$. Since (3.9) implies $d \lambda / d L>0$, it follows that $R_{0}>1$ if $L>L_{\text {crit }}$. That is, when the length of the stream is longer than the critical domain size, the population can persist in the stream.

Similar calculations show that the critical domain size under Danckwerts boundary conditions is given by

$$
L_{\text {crit }}^{\text {Dan }}=\frac{1}{\sqrt{-(a / 2 D)^{2}+(f-v) / D}}\left(\arctan \left(\frac{(a / D) \sqrt{-(a / 2 D)^{2}+(f-v) / D}}{(f-v) / D-(a / D)^{2} / 2}\right)+\pi\right) .
$$

Copyright $\odot$ by SIAM. Unauthorized reproduction of this article is prohibited. 


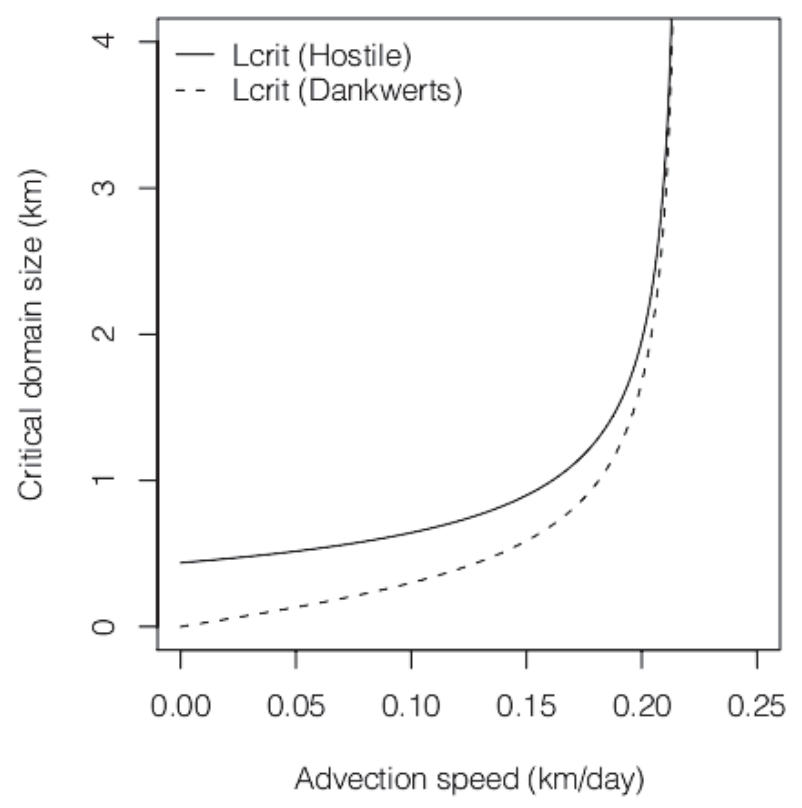

Figure 1. Comparison of the critical domain size required for persistence of a population described by (3.1) subject to hostile (solid) and Danckwerts (dashed) boundary conditions as a function of the advective speed a. The model parameters are $D=0.35 \mathrm{~m}^{2} / \mathrm{s}$ and $r=f-v=0.39 \mathrm{day}^{-1}$.

The relationship between domain length and stream flow is shown in Figure 1 for the case where $D=0.35 \mathrm{~m}^{2} / \mathrm{s}$ and $r=f-v=0.39$ day $^{-1}$. As stream flow (advection) increases, the critical domain size increases, eventually becoming infinite. As discussed in [20], longer domains are necessary for persistence when the boundary conditions are hostile, as compared to Danckwerts boundary conditions. This difference due to boundary conditions decreases as the stream flow increases.

We conclude this example with some numerical simulations to visualize the predicted relationship between the long-term behavior of the population model (2.7) and the next generation operator. Figures 2(a1) and 2(a2) show a population with an intrinsic growth rate of $r=0.39 \mathrm{~d}^{-1}$ being advected at $a=0.0015 \mathrm{~m} / \mathrm{s}$ along a $1 \mathrm{~km}$ river with hostile boundary conditions. The population persists when $D=0.35 \mathrm{~m}^{2} / \mathrm{s}$ and is washed out when $D=0.15$ $\mathrm{m}^{2} / \mathrm{s}$. The population model (2.7) was solved numerically using MATLAB's parabolic PDE solver pdepe. For comparison, Figures 2(b1) and 2(b2) show the action of the next generation operator $\Gamma$ on the dominant eigenfunction $\phi(x)$, which we approximated together with $R_{0}$ using the projection method described in section 2.4. We used the alternate definition of the next generation operator (Proposition 2.5) to compute $\Gamma \phi(x)$. For the case considered here where $a, D$ are constants, an exact expression for $k(x, y)$ exists (Figures 2(c1) and 2(c2), Appendix B), but the integration was done numerically. In each case, the persistence or washout of the population is predicted by $R_{0}$.

Example B: A model with variable birth rate. In this example we again assume $Q, A, D$ in (2.7) are constants but now allow the birth rate $f$ to vary in space. This is motivated by the observation that stream primary production increases with stream order as the stream 
(a1)

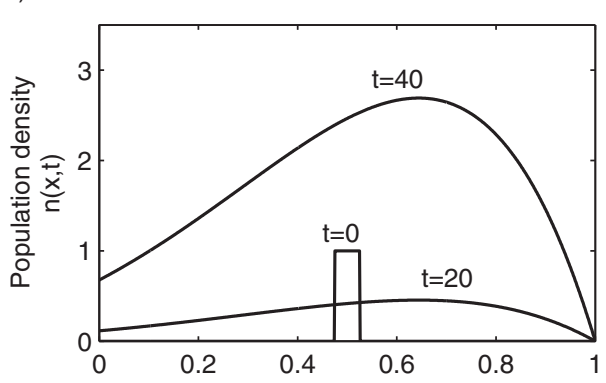

(b1)

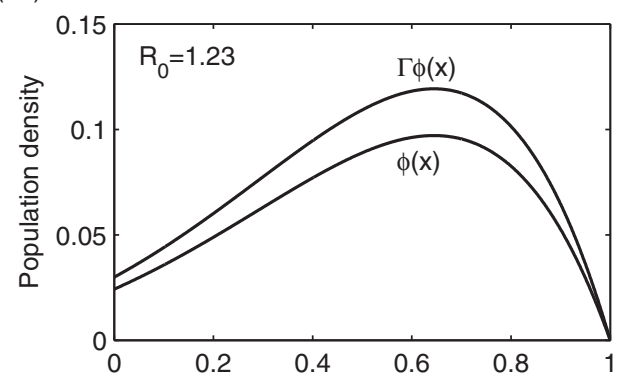

(c1)

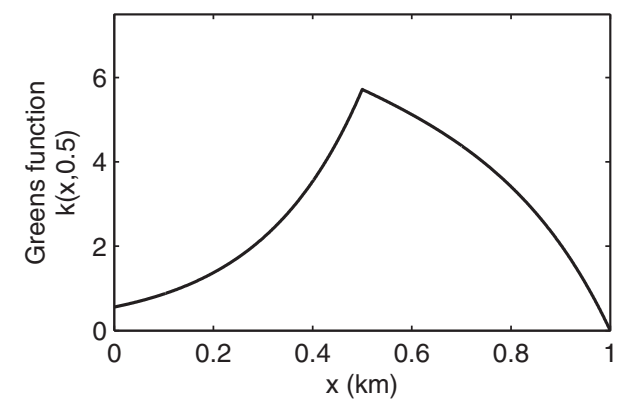

(a2)

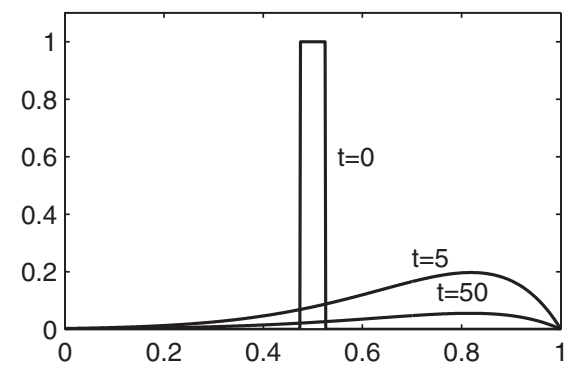

(b2)

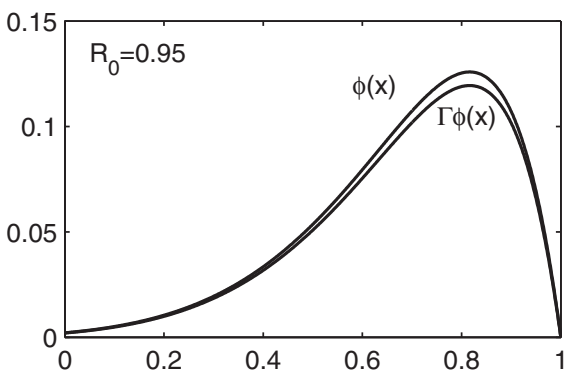

(c2)

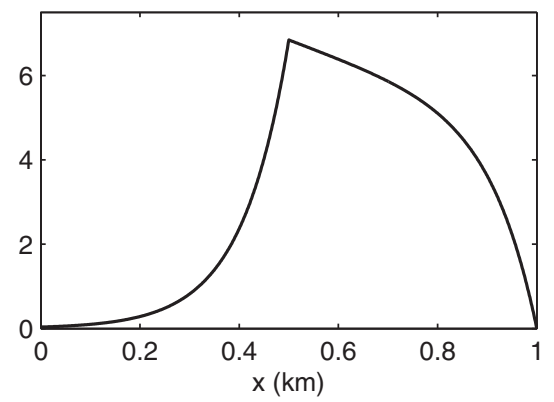

Figure 2. An exponentially growing population with an intrinsic growth rate of $r=0.39 d^{-1}$ being advected at $a=0.0015 \mathrm{~m} / \mathrm{s}$ along a $1 \mathrm{~km}$ river with hostile boundary conditions. Depending on the diffusion coefficient, the population either persists ((a1), $\left.D=0.35 \mathrm{~m}^{2} / \mathrm{s}\right)$ or washes out ((a2), $\left.D=0.15 \mathrm{~m}^{2} / \mathrm{s}\right)$ over time. The longterm behavior of the population is determined by the action of the next generation operator on the dominant eigenvector $\phi(\mathrm{b} 1),(\mathrm{b} 2) . \Gamma \phi(x)$ was computed using the alternate definition of $\Gamma$ and Green's function is shown in (c1), (c2).

widens and becomes less shaded [1]. For simplicity, we choose $f$ to be a linearly increasing function with distance downstream,

$$
f(x)=f_{\min }+\left(f_{\max }-f_{\min }\right)(x / L), \quad x \in[0, L]
$$

where $0 \leq f_{\min } \leq f_{\max }$ are the birth rates at the upstream and downstream boundaries of the stream, respectively. Under these assumptions, and considering Danckwert's boundary

Copyright (C) by SIAM. Unauthorized reproduction of this article is prohibited. 
conditions, (2.7) reduces to the linearization of the single-species model studied in [20],

$$
\begin{cases}n_{t}=(f(x)-v) n-a n_{x}+D n_{x x}, & x \in(0, L), t>0, \\ a n(0, t)-D n_{x}(0, t)=0, n_{x}(L, t)=0, & t>0, \\ n(x, 0)=n_{0}(x), & x \in(0, L) .\end{cases}
$$

Using the three measures of persistence, we study how the spatially variable birth rate, stream flow, and mortality rate affect population persistence. First, assuming a fixed mortality rate $v$, we compare population persistence in the case where the birth rate varies in space to the case where the birth rate is the constant average $f_{\text {avg }}=\left(f_{\min }+f_{\max }\right) / 2$. To do this we fix the growth rates $r_{\min }, r_{\max }$ and the mortality rate $v$ and from these compute $f_{\min }, f_{\max }$, and $f_{\text {avg. }}$.

We begin by describing the fundamental niche using $R_{\text {loc }}(x)$ for both the constant and variable birth rate environments. In the constant environment, $R_{\operatorname{loc}(x)}=f_{\text {avg }} / v$. Therefore, the entire stream is either suitable or unsuitable habitat. However, in the variable environment, $R_{\text {loc }}(x)=f(x) / v$. Since $f$ increases downstream, it is possible that even if the upstream habitat is unsuitable, there is suitable habitat downstream.

Next we consider the source-sink regions in the stream as modeled by $R_{\delta}(x)$. The results are shown in Figure 3 for three different mortality rates. When the birth rate is constant in space, the flow can result in the appearance of a sink region (where $R_{\delta}(x)<1$ ) associated with the downstream boundary condition (Figure 3(a1)-(a3)). The size of the sink region increases with increasing flow, as individuals are washed out more quickly and are unable to replace themselves in the next generation. This downstream sink region remains apparent when the birth rate is variable in space, although it is somewhat smaller in size as there is a trade-off with increasing birth rate downstream (Figures $3(\mathrm{c} 1)-(\mathrm{c} 3)$ ). In addition, there is a new upstream sink region associated with the reduced birth rate in the upstream region. This effect is mitigated to a certain extent with increasing flow, as individuals are carried further downstream and into better habitat during their lifetime and are therefore able to contribute more offspring to the next generation.

For the same population growth rates $r_{\min }$ and $r_{\max }$, different mortality rates $v$ lead to different source-sink regions. (Compare the solid, dashed, and dotted lines for each case in Figure 3.) As the mortality rate increases, $f_{\min }$ and $f_{\max }$ also increase since we assume that the growth rates $r_{\min }$ and $r_{\max }$ are constant. Therefore, increasing mortality rate reduces the size of the downstream sink region, as individuals complete their lifecycle more quickly and so are less subject to loss through the downstream boundary due to dispersal. This effect is stronger when the birth rate is constant in space. When the birth rate is variable in space, the effect of increasing mortality rate is countered by the increasing birth rate as individuals disperse downstream. This introduces a trade-off between living long enough to take advantage of the increased birth rates downstream, but not so long as to encounter the downstream boundary condition. The advantage conferred by high mortality in the case where birth rate is variable in space is most evident at medium flows, as individuals are more quickly dispersed downstream into more productive environments but are not washed out immediately, as is the case with the highest flow. This increased dispersal can be seen by considering Green's functions for low, medium, and high flows (Figures 3(b1)-(b3)). The interaction between flow and the spatially

Copyright (c) by SIAM. Unauthorized reproduction of this article is prohibited. 
(a1)

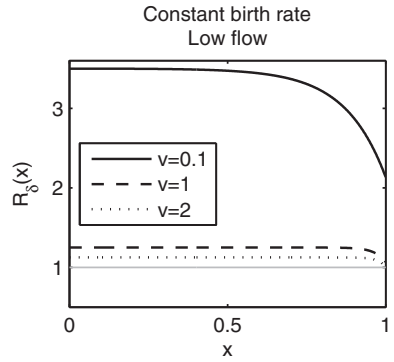

(a2)

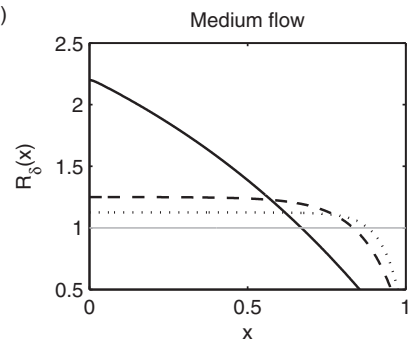

(a3)

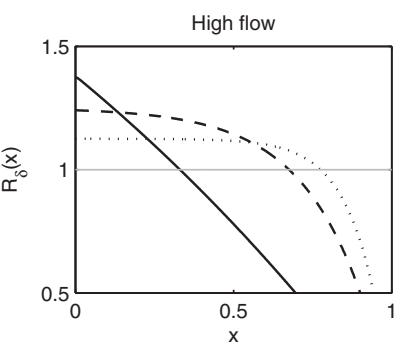

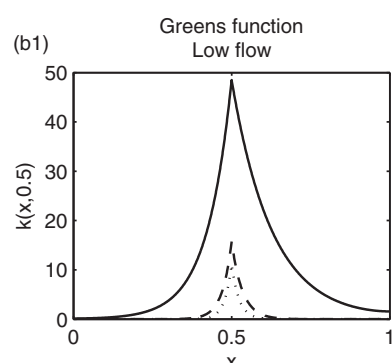
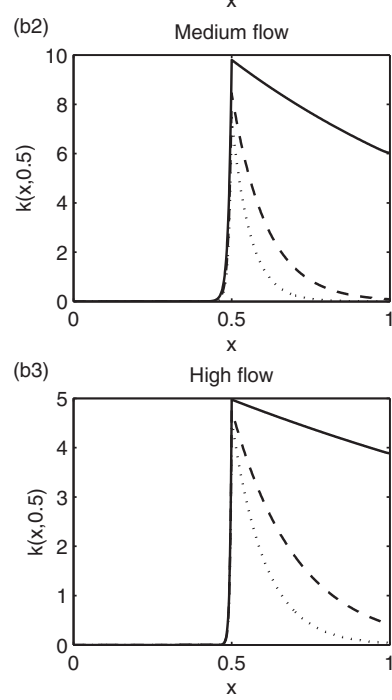
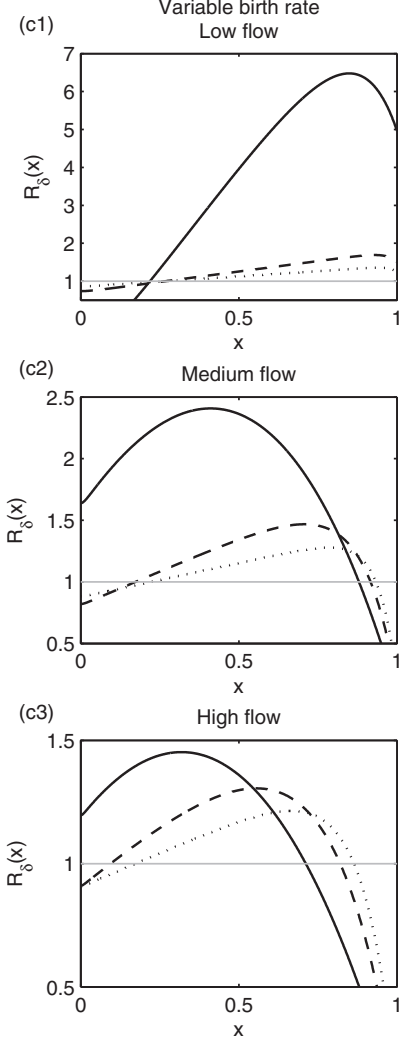

Figure 3. $R_{\delta}(x)$ for several flow speeds and mortality rates. When $f$ is constant (a1)-(a3) there is a negative downstream boundary effect created by the flow which reduces the size of the source region. This effect is mitigated when birth and mortality rates are increased. When $f(x)$ increases linearly with distance downstream (c1)-(c3), in addition to the downstream boundary effect caused by flow, the birth rate introduces a negative upstream boundary effect. This effect is increased when birth and mortality rates are high. In each case Green's functions are shown in (b1)-(b3). The other parameters are $r_{\text {avg }}=0.25, D=0.001, r_{\min }=-0.3, r_{\max }=0.8$.

variable growth rate suggests that there may be a range of flow speeds and mortality rates over which the proportion of the habitat that is a source is maximized.

To see more clearly how flow and mortality rate interact to influence the source-sink regions, we obtain the proportion of habitat for which $R_{\delta}(x)>1$ as a function of flow speed and mortality rate in a habitat with constant birth rate (Figure 4(a1)) and spatially variable birth rate (Figure 4(b1)). In both habitats, the effect of the interaction between mortality rate and flow on the size of the source region is evident over a range of values. However, using $R_{\delta}(x)$ as a measure of persistence we are not able to determine how the global population persistence is affected. For this we turn to $R_{0}$.

For the same range of flow speeds and mortality rates as in Figures 4(a1) and 4(b1), we calculated $R_{0}$ using the method as described in section 2 (see also [6]) with $\mathrm{n}=100$. The results are shown in Figures 4(a2) and 4(b2). Similar patterns of $R_{0}$ as a function of stream flow and mortality rate are apparent in both the constant and variable birth rate cases. $R_{0}$ is highest for low values of stream flow and mortality rate, and decreases with 

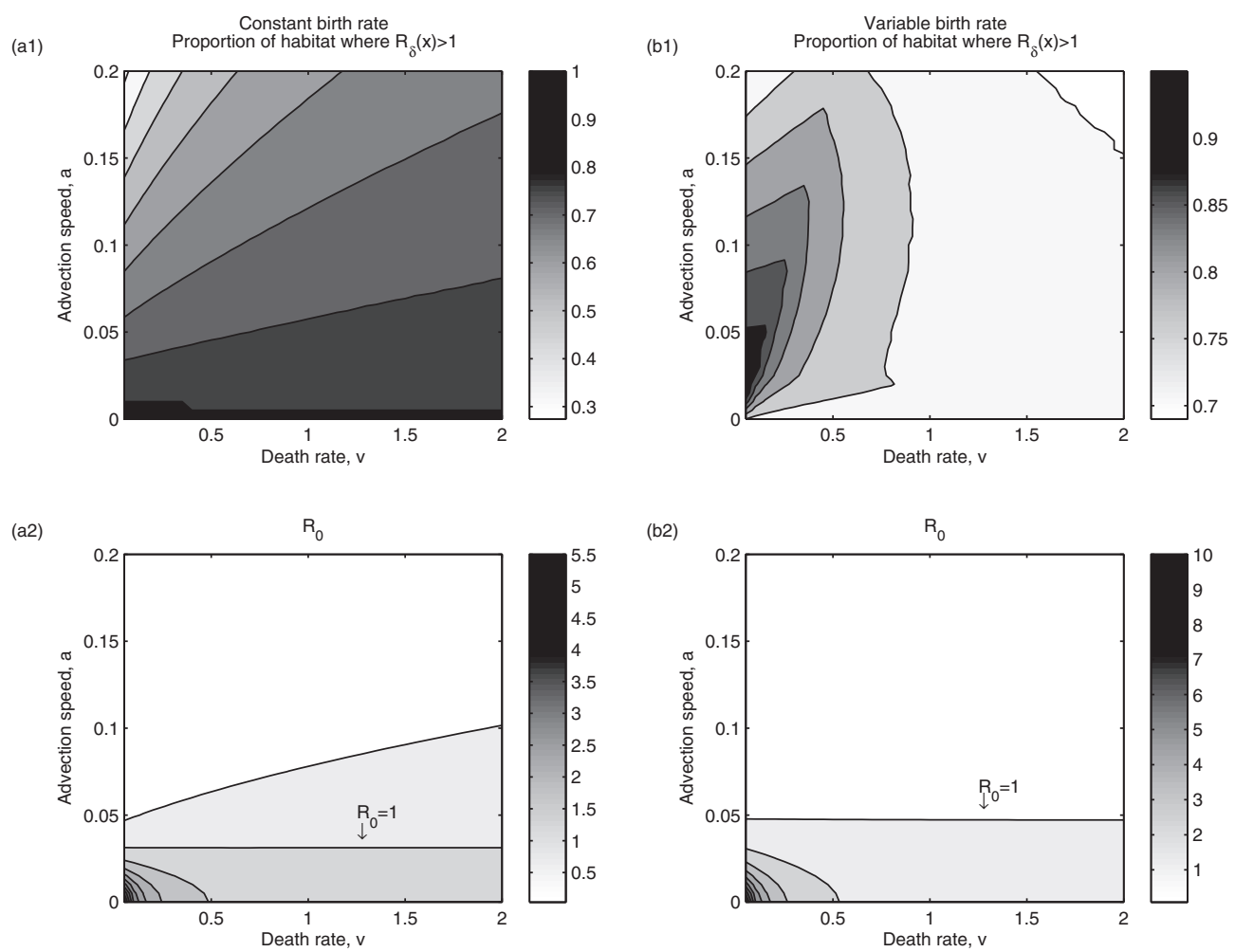

Figure 4. The proportion of habitat where $R_{\delta}(x)>1$ for a range of flow speeds and mortality rates for the case where $f$ is constant (a1) and linearly increasing (b1). The corresponding plots of $R_{0}$ are shown in (a2), (b2). The other parameters are $r_{\text {avg }}=0.25, D=0.001, r_{\min }=-0.3, r_{\max }=0.8$.

increasing flow and mortality. The influence of mortality rates in mitigating the effect of the downstream boundary observed in Figures 4(a1) and 4(b1) does not translate to increased global persistence for higher mortality rates. In fact, in the constant environment simulations, the advection speed corresponding to the threshold where $R_{0}=1$ is approximately 0.03 and remains constant for all mortality rates (Figure 4(a2)). This value is very similar to the predicted spreading speed $c^{*}=2 D(f-v)=0.032$ for the population in the absence of advection. The advection speed permitting persistence $\left(R_{0}>1\right)$ in the variable environment is higher than in the constant environment, suggesting that the spatial variability in birth rate, in particular the higher birth rate near the downstream boundary, permits persistence under increased stream flow as compared to the constant environment case.

In comparing the results in Figures 4(a1) and 4(b1) with Figures 4(a2) and 4(b2), we further see that it is possible to have $R_{0}>1$ even when the proportion of the domain where $R_{\delta}(x)>1$ is less than 1 . This indicates that it is not necessary for the entire, or even the majority, of the domain, to be a source in order for the population to persist.

4. Discussion. In this paper we considered persistence of populations in streams described by the advection-diffusion-reaction model (2.1) using the three measures of persistence proposed by [16] in the context of a next generation operator. We formulated the next generation 
operator for the linearization of (2.1) and used this to derive the three measures of persistence for our model. In particular, we defined the net reproductive rate $R_{0}$ and used the results of [30] to prove that $R_{0}$ determines the stability of the trivial homogeneous steady state of the model and therefore whether a population is able to invade and persist in the river. Using this new perspective on population persistence in streams we revisited a model in [26] and obtained results consistent with previous critical domain size analysis [26, 17]. In addition, we used these measures of persistence to study a model where birth rate increased linearly downstream, which provided new insight into the interaction between flow speed and mortality rate and their effects on population persistence.

The previous theoretical work in [30] established the connection between the stability of the trivial steady state solution and $R_{0}$ for a very general class of models. However, the infinite-dimensional examples in [30] concern populations which are continuous in time with a continuous age structure. In contrast, we studied a model where the population was continuous with a continuous spatial distribution, where dispersal is represented by a very general linear operator. Although we formulated the next generation operator starting from an advectiondiffusion-reaction population model, these ideas suggest a more general approach for defining next generation operators. For example, recall the alternate definition of the next generation operator,

$$
\Gamma \psi(x)=f(x) \int_{0}^{L} k(x, y) \psi(y) d y,
$$

where $k(x, y)$ represents the lifetime density of space use of an individual initially introduced at $y$. In this case $k$ was derived from the associated population model, (2.7); however, $k$ could be derived from other sources, such as numerical simulations of river flow, which include additional hydrodynamic complexity (e.g., River2D by [28]).

In Example $\mathrm{B}$ when the environment was variable we found by using $R_{\delta}(x)$ that both the upstream boundary and the downstream boundary affected persistence. In the case of the downstream boundary the effect was due to the loss of individuals and the strength of the effect was strongly connected to the lifetime of the individual and the flow. When the spatial scale of the river is much longer than the lifetime of the organism, then the effect of the downstream boundary on $R_{\delta}(x)$ will be restricted to initial locations that are close to the boundary. However, for organisms that have long lifetimes compared to the spatial scale of the river, this effect will be significant as most individuals, regardless of starting location, will interact with the boundary during their lifetime. Therefore, the downstream boundary effect has to do with the interaction between flow speed and lifetime. In contrast, the upstream boundary effect is due to the negative growth rate associated with poor primary productivity in the upper reaches of the stream. In this case, flow speed mitigates the effect, as it enables organisms to be carried downstream to better habitat. This introduces a trade-off between low mortality rates, which reduce the effect of the upstream boundary, and high mortality rates, which reduce the effect of the downstream boundary. It would be interesting to do a meta-analysis of the lifespan of aquatic organisms compared to the spatial scale of the reaches in which they are found to see if there is a relationship.

A natural extension of the theory developed here for studying a single population using the measures of persistence would be to develop the theory necessary to study more general

Copyright (c) by SIAM. Unauthorized reproduction of this article is prohibited. 
models for interacting and structured populations. This would enable $R_{0}$ analysis of models such as the benthic-drift model of [22],

$$
\begin{aligned}
& \frac{\partial n_{d}}{\partial t}=\mu n_{b}-\sigma n_{d}+\mathcal{L} n_{d}, \\
& \frac{\partial n_{b}}{\partial t}=g\left(n_{b}\right) n_{b}-\mu n_{b}+\sigma n_{d}
\end{aligned}
$$

where $n_{b}$ is the population density on the benthos, $n_{d}$ is the population density in the drift, and $\mu, \sigma$ are the rates of entering and leaving the drift, respectively, or the competition model of $[20]$,

$$
\begin{aligned}
& \frac{\partial n_{1}}{\partial t}=n_{1}\left(r_{1}(x)-A_{11} n_{1}-A_{12} n_{2}\right)+\mathcal{L} n_{1} \\
& \frac{\partial n_{2}}{\partial t}=n_{2}\left(r_{2}(x)-A_{22} n_{2}-A_{21} n_{1}\right)+\mathcal{L} n_{2}
\end{aligned}
$$

where $n_{1}, n_{2}$ are the densities of the two competition species, $r_{i}$ are their respective growth rates, and $A_{i j}$ are the intra- and interspecific competition coefficients. Analysis of structured population models often requires the study of limiting cases (e.g., [22]) or numerical approximations (e.g., [20]). However, previous application of $R_{0}$ analysis to other types of structured population models has yielded insight into the effect of parameters on persistence not possible from direct analysis of the population model [8]. Therefore, $R_{0}$ analysis of models of structured populations in streams may prove fruitful.

It is our hope that the theory developed here will provide a foundation for further development of a similar theory for the more complex population models mentioned above. In addition, we hope to encourage the connection of data to these models in order to further understand their potential as management tools for assessing persistence of populations in streams under varying flow conditions.

Appendix A. Proofs. Recall that $X=C[0, L]$ denotes the Banach space of continuous functions on the interval $[0, L]$ with the supremum norm $\|u\|_{\infty}=\max _{x \in[0, L]}|u(x)|$ and $X_{+}=$ $\{f \in X: f(x) \geq 0$ for all $x \in[0, L]\}$ denotes the cone of nonnegative functions in $X$.

\section{A.1. Proof of Proposition 2.2.}

Proof. Let $\mu_{1}>0$ be the principal eigenvalue of $(2.8)$ and $\hat{n}(x)$ the equilibrium solution of (2.1) as defined in Proposition 2.1.

Case 1. When the boundary conditions in (2.1) are Neumann or Robin type (i.e., $\beta_{1} \neq 0$ and $\left.\beta_{2} \neq 0\right)$, any solution of (2.1) with nonnegative and nonzero initial data $n(x, 0)$ satisfies $n(x, t)>0$ on $[0, L]$, so $\hat{n}(x)>0$ for all $x \in[0, L]$. In particular, if we let $\delta=\frac{1}{2} \min _{x \in[0, L]} \hat{n}(x)$, then it follows that $\delta>0$. If $n(x, t)$ is any solution of $(2.1)$ with $n(x, 0)=n_{0} \in X_{+} \backslash\{0\}$, then it follows from Proposition 2.1(ii) that eventually $n(x, t) \geq \bar{n}(x, t)$, where $\bar{n}(x, t)$ is a solution of (2.1) satisfying $\bar{n}(x, t) \rightarrow \hat{n}(x)$ as $t \rightarrow \infty$. Thus $\liminf _{t \rightarrow \infty} n(x, t) \geq \liminf _{t \rightarrow \infty} \bar{n}(x, t)$ uniformly for $x \in[0, L]$ and

$$
\liminf _{t \rightarrow \infty}(n(x, t)-\delta) \geq \liminf _{t \rightarrow \infty}(\bar{n}(x, t)-\delta) \geq \liminf _{t \rightarrow \infty}(\bar{n}(x, t)-\hat{n}(x))=0
$$

Copyright (C) by SIAM. Unauthorized reproduction of this article is prohibited. 
uniformly for $x \in[0, L]$. Therefore,

$$
\liminf _{t \rightarrow \infty} n(x, t) \geq \delta \text { uniformly for } x \in[0, L]
$$

and hence, $\liminf _{t \rightarrow \infty} \min _{x \in[0, L]} n(x, t) \geq \delta>0$.

Case 2. When at least one of the boundary conditions in (2.1) is Dirichlet type (i.e., $\beta_{1}=0$ or $\beta_{2}=0$ ), we have $\hat{n}(0)=0$ or $\hat{n}(L)=0$ (or possibly both) but $\hat{n} \not \equiv 0$. Let $\delta=\frac{1}{L} \int_{0}^{L} \hat{n}(x) d x$. Then $\delta>0$. Similar to Case 1, for any solution $n(x, t)$ of $(2.1)$ with $n(x, 0)=n_{0} \in X_{+} \backslash\{0\}$, there exists some solution $\bar{n}(x, t)$ of $(2.1)$ with $\bar{n}(x, t) \rightarrow \hat{n}(x)$ as $t \rightarrow \infty$ uniformly for $x \in[0, L]$ such that $n(x, t) \geq \bar{n}(x, t)$ for all $x \in[0, L]$ and for sufficiently large $t>0$. Then $\lim _{t \rightarrow \infty}\left(\max _{x \in[0, L]} \bar{n}(x, t)-\max _{x \in[0, L]} \hat{n}(x)\right)=0$, and hence,

$$
\begin{aligned}
\liminf _{t \rightarrow \infty}\left(\max _{x \in[0, L]} n(x, t)-\delta\right) & \geq \liminf _{t \rightarrow \infty}\left(\max _{x \in[0, L]} n(x, t)-\max _{x \in[0, L]} \hat{n}(x)\right) \\
& \geq \liminf _{t \rightarrow \infty}\left(\max _{x \in[0, L]} \bar{n}(x, t)-\max _{x \in[0, L]} \hat{n}(x)\right) \\
& =\lim _{t \rightarrow \infty}\left(\max _{x \in[0, L]} \bar{n}(x, t)-\max _{x \in[0, L]} \hat{n}(x)\right)=0 .
\end{aligned}
$$

Therefore, $\liminf \operatorname{in}_{t \rightarrow \infty} \max _{x \in[0, L]} n(x, t) \geq \delta$.

\section{A.2. Proof of Proposition 2.4.}

Proof. Let $\psi_{0} \in X$ and $\psi$ be the solution to (2.11) with initial function $\psi_{0}$. Clearly, $\Gamma \psi_{0}$ is defined on $[0, L]$. Let $\underline{v}=\min _{x \in[0, L]} v(x)$ (recall $v(x)>0$ is the mortality rate). Then $w(x, t)=e^{\underline{v} t} \psi(x, t)$ satisfies

$$
\begin{cases}w_{t}=\underline{v} w-v(x) w+\mathcal{L} w, & x \in(0, L), t>0, \\ w(x, 0)=\psi_{0}(x), & x \in(0, L),\end{cases}
$$

subject to the same boundary conditions as $\psi$.

Claim. $|w(x, t)| \leq\left\|\psi_{0}\right\|_{\infty}$ for all $x \in[0, L]$ and $t>0$.

In fact, by the weak parabolic maximum principle for (A.1) the maximum of $w(x, t)$ occurs on the parabolic boundary (i.e., either on the boundary of $[0, L]$ or at the initial time $t=0$; see, e.g., [4, Theorem 1.17]). Consider first the boundary condition at $x=0$. If it is of Dirichlet type $\left(\alpha_{1}=1, \beta_{1}=0\right)$, then $w(0, t)=0$ for all $t>0$ and the maximum cannot occur at $x=0$ unless $w(x) \equiv 0$. If it is of Neumann or Robin type, $\left(\alpha_{1} \geq 0, \beta_{1}>0\right)$, then the boundary condition at $x=0$ implies that $w_{x}(0, t)=\frac{\alpha_{1}}{\beta_{1}} w(0, t) \geq 0$ for all $t>0$, since $w(0, t) \geq 0$ for all $t>0$ by the comparison principle. Therefore the maximum of $w(x, t)$ cannot occur at $x=0$ unless $w(x, t) \equiv 0$. By similar arguments we find that the maximum of $w(x, t)$ is not attained at $x=L$. Since the maximum is not attained on the boundary of $[0, L]$, it must be attained at $t=0$, and so $w(x, t) \leq w(x, 0)=\psi_{0}(x) \leq\left\|\psi_{0}\right\|_{\infty}$ for all $x \in[0, L]$ and $t>0$, proving the claim.

Copyright (c) by SIAM. Unauthorized reproduction of this article is prohibited. 
Now we show $\Gamma \psi_{0} \in X$. Let $x, y \in[0, L]$. Then

$$
\begin{aligned}
\left|\Gamma \psi_{0}(x)-\Gamma \psi_{0}(y)\right|= & \left|f(x) \int_{0}^{\infty} w(x, t) e^{-\underline{v} t} d t-f(y) \int_{0}^{\infty} w(y, t) e^{-\underline{v} t} d t\right| \\
\leq & \left|f(x) \int_{0}^{\infty}(w(x, t)-w(y, t)) e^{-\underline{v} t} d t\right| \\
& +|f(x)-f(y)| \int_{0}^{\infty} w(y, t) e^{-\underline{v} t} d t \\
\leq & \|f\|_{\infty} \int_{0}^{\infty}|w(x, t)-w(y, t)| e^{-\underline{v} t} d t+|f(x)-f(y)| \cdot \frac{\left\|\psi_{0}\right\|_{\infty}}{\underline{v}} .
\end{aligned}
$$

For any $\epsilon>0$, it follows from the continuity of $f$ that there exists $\delta_{1}>0$ such that $\mid f(x)-$ $f(y) \mid<\epsilon \underline{v} /\left(2\left\|\psi_{0}\right\|_{\infty}\right)$ for any $x, y \in[0, L]$ with $|x-y|<\delta_{1}$. Let $T$ be sufficiently large so that $2\left\|\psi_{0}\right\|_{\infty} e^{-\underline{v} T} / \underline{v}<\epsilon /\left(4\|f\|_{\infty}\right)$. By the continuity of $w(x, t)$ we can find a $\delta_{T}>0$ such that $|w(x, t)-w(y, t)|<\epsilon \underline{v} /\left(4\|f\|_{\infty}\left(1-e^{-\underline{v} T}\right)\right)$ for any $x, y \in[0, L]$ with $|x-y|<\delta_{T}$ and $t \in[0, T]$. Let $\delta=\min \left\{\delta_{1}, \delta_{T}\right\}$. Then for any $x, y \in[0, L]$ with $|x-y|<\delta$, we have

$$
\begin{aligned}
\|f\|_{\infty} \int_{0}^{\infty}|w(x, t)-w(y, t)| e^{-\underline{v} t} d t= & \|f\|_{\infty} \int_{0}^{T}|w(x, t)-w(y, t)| e^{-\underline{v} t} d t \\
& +\|f\|_{\infty} \int_{T}^{\infty}|w(x, t)-w(y, t)| e^{-\underline{v} t} d t \\
& <\|f\|_{\infty} \frac{\epsilon \underline{v}}{4\|f\|_{\infty}\left(1-e^{-\underline{v} T}\right)} \int_{0}^{T} e^{-\underline{v} t} d t \\
& +\|f\|_{\infty} 2\left\|\psi_{0}\right\|_{\infty} \int_{T}^{\infty} e^{-\underline{v} t} d t \\
& <\frac{\epsilon}{4}+\frac{\epsilon}{4} \\
& <\frac{\epsilon}{2} .
\end{aligned}
$$

Thus for $|x-y|<\delta$ we have

$$
\left|\Gamma \psi_{0}(x)-\Gamma \psi_{0}(y)\right|<\frac{\epsilon}{2}+\frac{\epsilon}{2}=\epsilon,
$$

which proves $\Gamma \psi_{0}$ is continuous on $[0, L]$ and hence $\Gamma \psi_{0} \in X$.

\section{A.3. Proof of Proposition 2.5.}

Proof. First consider the case $f(x)=1$. In this case, the next generation operator associated with $(2.7)$ is

$$
\bar{\Gamma} \psi_{0}(x)=\int_{0}^{\infty} \psi(x, t) d t
$$

where $\psi_{0} \in X$ and $\psi(x, t)$ is the solution to (2.11) subject to the same boundary conditions as (2.7). It follows from Proposition 2.4 that $\bar{\Gamma}: X \rightarrow X$ is well defined. We claim $\bar{\Gamma}$ is equivalent 
to

$$
\bar{\Gamma} \psi_{0}(x)=\int_{0}^{L} k(x, y) \psi_{0}(y) d y
$$

where $k(x, y)$ is the solution for a fixed value of $y$ of the equation

$$
-v(x) k(x, y)+\mathcal{L} k(x, y)=-\delta(x-y)
$$

subject to the boundary conditions $\alpha_{1} k(0, y)-\beta_{1} k^{\prime}(0, y)=0, \alpha_{2} k(L, y)+\beta_{2} k^{\prime}(L, y)=0$, where ' denotes differentiation with respect to $x$. This can be seen by integrating the differential equation in (2.11) with respect to $t$ from 0 to $\infty$ to obtain

$$
\int_{0}^{\infty} \psi_{t}(x, t) d t=-v(x) \int_{0}^{\infty} \psi(x, t) d t+\mathcal{L} \int_{0}^{\infty} \psi(x, t) d t .
$$

Recognizing $\bar{\Gamma} \psi_{0}(x)$ in the terms on the right-hand side and using the fact that $\psi(x, t) \rightarrow 0$ as $t \rightarrow \infty$ for all $x \in[0, L]$, we obtain the following boundary value problem for $\bar{\Gamma} \psi_{0}(x)$ :

$$
\left\{\begin{array}{l}
-v(x) \bar{\Gamma} \psi_{0}(x)+\mathcal{L}\left[\bar{\Gamma} \psi_{0}\right](x)=-\psi_{0}(x), \quad x \in(0, L), \\
\alpha_{1} \bar{\Gamma} \psi_{0}(0)-\beta_{1}\left(\bar{\Gamma} \psi_{0}\right)^{\prime}(0)=0, \\
\alpha_{2} \bar{\Gamma} \psi_{0}(L)+\beta_{2}\left(\bar{\Gamma} \psi_{0}\right)^{\prime}(L)=0 .
\end{array}\right.
$$

The boundary conditions for $\bar{\Gamma}$ are obtained from (A.2) and the boundary conditions for $\psi(x, t)$. For homogeneous boundary conditions such as those considered here, the solution of (A.5) can be expressed as

$$
\bar{\Gamma} \psi_{0}(x)=\int_{0}^{L} k(x, y) \psi_{0}(y) d y
$$

where $k(x, y)$ is the Green's function associated with (A.5) as defined by the solution of

$$
\left\{\begin{array}{l}
-v(x) k(x, y)+\mathcal{L} k(x, y)=-\delta(x-y), \quad x \in(0, L), \\
\alpha_{1} k(0, y)-\beta_{1} k^{\prime}(0, y)=0 \\
\alpha_{2} k(L, y)+\beta_{2} k^{\prime}(L, y)=0
\end{array}\right.
$$

for a fixed $y \in(0, L)$, where ' denotes differentiation with respect to $x$ (see, e.g., [27]).

For the general case where $f=f(x)$ we have

$$
\Gamma \psi_{0}(x)=f(x) \int_{0}^{\infty} \psi(x, t) d t=f(x) \bar{\Gamma} \psi_{0}(x)=f(x) \int_{0}^{L} k(x, y) \psi_{0}(y) d y
$$

\section{A.4. Proof of Proposition 2.6.}

Proof. Let $\psi_{0}, \phi_{0} \in X$. The linearity of $\Gamma$ follows from the linearity of the partial differential equations for $\psi(x, t)$ and $\phi(x, t)$, and the linear homogeneous boundary conditions. Note 
that since $k(x, y)$ is continuous on $[0, L] \times[0, L]$, there exists $M>0$ such that $|k(x, z)|<M$ for all $x, z \in[0, L]$. To show that $\Gamma$ is bounded, let $\psi_{0} \in X$. Then

$$
\left\|\Gamma \psi_{0}\right\|_{\infty}=\max _{x \in[0, L]}\left|\Gamma \psi_{0}(x)\right|=\max _{x \in[0, L]}\left|f(x) \int_{0}^{L} k(x, y) \psi_{0}(y) d y\right| \leq M L\|f\|_{\infty}\left\|\psi_{0}\right\|_{\infty} .
$$

Therefore $\|\Gamma\|_{*} \leq M L\|f\|_{\infty}$, where $\|\cdot\|_{*}$ is the operator norm in $C(X, X)$, and so $\Gamma$ is a bounded operator. To show that $\Gamma$ is compact, let $\left\{u_{k}\right\}_{k=1}^{\infty} \subset X$ be a bounded sequence. Then there exists a constant $M_{1}>0$ such that $\left\|u_{k}\right\|_{\infty} \leq M_{1}$ for all $k \in \mathbb{N}$. Therefore $\left\{\Gamma u_{k}\right\}_{k=1}^{\infty} \subset X$ is bounded by $\frac{\|f\|_{\infty} M_{1}}{v(x)}$ because $\Gamma$ is a bounded operator with $\|\Gamma\|_{*} \leq \frac{\|f\|_{\infty}}{v(x)}$. We now show that $\left\{\Gamma u_{k}\right\}_{k=1}^{\infty}$ is equicontinuous on $[0, L]$. For any $x, y \in[0, L]$ and $k \in \mathbb{N}$, we have

$$
\begin{aligned}
\left|\Gamma u_{k}(x)-\Gamma u_{k}(y)\right|= & \left|f(x) \int_{0}^{L} k(x, z) u_{k}(z) d z-f(y) \int_{0}^{L} k(y, z) u_{k}(z) d z\right| \\
\leq & \left|f(x) \int_{0}^{L} k(x, z) u_{k}(z) d z-f(y) \int_{0}^{L} k(x, z) u_{k}(z) d z\right| \\
& +\left|f(y) \int_{0}^{L} k(x, z) u_{k}(z) d z-f(y) \int_{0}^{L} k(y, z) u_{k}(z) d z\right| \\
\leq & |f(x)-f(y)| \int_{0}^{L}\left|k(x, z) u_{k}(z)\right| d z+|f(y)| \int_{0}^{L}\left|k(x, z)-k(y, z) u_{k}(z)\right| d z \\
\leq & M_{1}|f(x)-f(y)| \int_{0}^{L}|k(x, z)| d z+M_{1}\|f\|_{\infty} \int_{0}^{L}|k(x, z)-k(y, z)| d z .
\end{aligned}
$$

Let $\epsilon>0$ be given. Since $f$ is continuous on $[0, L]$, there exists $\delta_{1}>0$ such that $|f(x)-f(y)|<$ $\epsilon /\left(2 M_{1} M_{2} L\right)$ for all $x, y \in[0, L]$ with $|x-y|<\delta_{1}$. Moreover, there exists $\delta_{2}>0$ such that $|k(x, z)-k(y, z)|<\epsilon /\left(2 M_{1} L\|f\|_{\infty}\right)$ for $x, y \in[0, L]$ with $|x-y|<\delta_{2}$, uniformly for $z \in[0, L]$. Let $\delta=\min \left\{\delta_{1}, \delta_{2}\right\}$. Then for any $k \in \mathbb{N}$ and $x, y \in[0, L]$ with $|x-y|<\delta$, we have

$$
\begin{aligned}
\left|\Gamma u_{k}(x)-\Gamma u_{k}(y)\right| & \leq M_{1} \frac{\epsilon}{2 L M_{1} M_{2}} \int_{0}^{L} M_{2} d z+M_{1}\|f\|_{\infty} \int_{0}^{L} \frac{\epsilon}{2 M_{1} L\|f\|_{\infty}} d z \\
& =\epsilon / 2+\epsilon / 2 \\
& =\epsilon
\end{aligned}
$$

Therefore the bounded sequence $\left\{\Gamma u_{k}\right\}_{k=1}^{\infty} \subset X$ is also equicontinuous and by the ArzelàAscoli theorem (see, e.g., [14]), it follows that the sequence $\left\{\Gamma u_{k}\right\}_{k=1}^{\infty} \subset X$ has a convergent subsequence in $X$ and hence $\Gamma$ is a compact operator.

\section{A.5. Proof of Proposition 2.8.}

Proof. First we consider the case that both boundary conditions (upstream and downstream) are Neumann or Robin boundary conditions. In this case the result follows from the Krein-Rutman theorem once we establish that $\Gamma$ is a strongly positive compact linear operator. Note that the set of nonnegative functions forms a solid cone $X_{+}$in the Banach space $X$ with interior $\operatorname{Int}\left(X_{+}\right)=\{f \in X: f>0$ for all $x \in[0, L]\}$. For any $\psi_{0} \in X_{+} \backslash\{0\}$, we 
have $\Gamma \psi_{0}(x)=f(x) \int_{0}^{\infty} \psi(x, t) d t$, where $\psi(x, t)$ satisfies (2.11). By the comparison theorem, $\psi(x, t) \geq 0$ for all $t>0$ and $\psi \not \equiv 0$. Since $f(x)>0$ for all $x \in[0, L]$ we have $\Gamma \psi_{0}(x)>0$ for all $x \in[0, L]$, and hence $\Gamma \psi_{0} \in \operatorname{Int}\left(X_{+}\right)$. Therefore, $\Gamma$ is strongly positive. The linearity and compactness of $\Gamma$ have been proved in Proposition 2.6. Then the proposition follows from the Krein-Rutman theorem (Theorem 2.7).

When one or more boundary conditions are Dirichlet, then the solutions can vanish on the boundary and hence will not lie in the interior of $X_{+}$. In this case, the Krein-Rutman theorem on the space $X$ does not apply because $\Gamma$ is not strongly positive on $X$. To address this situation we use the approach in [25] (see, e.g., Chapter 7, Corollary 4.2) to define a space where $\Gamma$ is strongly positive.

Let $\bar{X}=C_{0}[0, L]$ denote the Banach space of continuous functions on $[0, L]$ vanishing on the boundary, $\bar{X}_{+}$the cone of nonnegative functions in $\bar{X}$, and $U$ the closed subspace of $C^{1}[0, L]$ consisting of continuously differentiable functions vanishing on the boundary. The set $U_{+}=U \cap \bar{X}_{+}$is a solid cone in $U$. Define the partial order $\ll$ on $U$ by $u_{1} \ll u_{2}$ provided $u_{2}-u_{1} \in \operatorname{Int}\left(U_{+}\right)$. Then by Corollary 4.2 in [25], we know that (2.7) defines a semiflow $\left\{\Phi_{t}\right\}_{t \geq 0}$ that satisfies $\Phi_{t}$ is continuous from $\bar{X}$ to $U$ and is strongly order preserving for each $t>0$. That is, for any $\phi, \psi \in \bar{X}_{+}$with $\phi<\psi$, we have $\Phi_{t}(\phi) \ll \Phi(\psi)$ for all $t>0$. Now, arguing as the previous case, one can prove that $\Gamma$ is strongly positive on $U$. Following similar arguments as in Proposition 2.6, one also obtains the linearity and compactness of $\Gamma$ on $U$. Thus, by applying the Krein-Rutman theorem to $\Gamma$ on $U$, we complete the proof in the case that at least one of the boundary conditions is a Dirichlet boundary condition.

\section{A.6. Proof of Proposition 2.10.}

Proof. We claim that $B^{-1}: X \rightarrow Z$ is defined by $B^{-1} u(x)=-\frac{1}{f(x)} \Gamma u(x)$. To prove this we will show that $B B^{-1}=I$ and $B^{-1} B=I$. Let $u \in X$ and $U(x, t)$ be the solution of (2.11) with initial function $u$. We have

$$
\begin{aligned}
B B^{-1} u(x) & =B\left(-\int_{0}^{\infty} U(x, t) d t\right) \\
& =-v(x)\left(-\int_{0}^{\infty} U(x, t) d t\right)+\mathcal{L}\left(-\int_{0}^{\infty} U(x, t) d t\right) \\
& =\int_{0}^{\infty} v(x) U(x, t)-\mathcal{L} U(x, t) d t \\
& =-\int_{0}^{\infty} U_{t}(x, t) d t \\
& =-\lim _{T \rightarrow \infty} U(x, T)+U(x, 0) \\
& =u(x),
\end{aligned}
$$

where we used (2.11) and the fact that $\lim _{T \rightarrow \infty} U(x, T)=0$. Conversely, for $w \in Z$,

$$
B^{-1} B w(x)=B^{-1}(-v(x) w(x)+\mathcal{L} w(x))=-\int_{0}^{\infty} W(x, t) d t
$$

Copyright (c) by SIAM. Unauthorized reproduction of this article is prohibited. 
where $W(x, t)$ satisfies

$$
\begin{cases}W_{t}=-v(x) W+\mathcal{L} W, & x \in[0, L], t>0, \\ W(x, 0)=-v(x) w(x)+\mathcal{L} w(x), & x \in(0, L),\end{cases}
$$

together with the appropriate boundary conditions. Let $\phi(x)=-\int_{0}^{\infty} W(x, t) d t$ for $x \in[0, L]$. We will show that $\phi(x)=w(x)$. In fact,

$$
-v(x) \phi(x)+\mathcal{L} \phi(x)=\int_{0}^{\infty} v(x) W-\mathcal{L} W d t=\int_{0}^{\infty}-W_{t} d t=-v(x) w(x)+\mathcal{L} w(x),
$$

since $\lim _{T \rightarrow \infty} W(x, T)=0$. In addition, $\alpha_{1} \phi(0)-\beta_{1} \phi^{\prime}(0)=0$ and $\alpha_{2} \phi(L)+\beta_{2} \phi^{\prime}(L)=0$ from the definition of $\phi(x)$ and the boundary conditions on $W(x, t)$. This implies that $\psi(x)=$ $\phi(x)-w(x)$ solves the boundary value problem

$$
\left\{\begin{array}{l}
\mathcal{L} \psi-v(x) \psi=0, \quad x \in(0, L), \\
\alpha_{1} \psi(0)-\beta_{1} \psi^{\prime}(0)=0, \\
\alpha_{2} \psi(L)+\beta_{2} \psi^{\prime}(L)=0 .
\end{array}\right.
$$

Since $\mathcal{L}$ is elliptic and $v(x)>0$, this admits only the trivial solution $\psi=0$ (see, e.g., [4, Theorem 1.16]), and hence $\phi=w$ and $B^{-1} B=I$ as well. Therefore, $\Gamma \psi_{0}(x)=f(x) \int_{0}^{\infty} \psi(x, t) d t=$ $\left[-C B^{-1} \psi_{0}\right](x)$, where $C w(x)=f(x) w(x)$.

To prove the spectral bound of $A=B+C$ has the same sign as $R_{0}$ (the spectral radius of $\Gamma=-C B^{-1}$ ) we first prove $s(B)<0$. For this, note that for the eigenvalue problem

$$
\left\{\begin{array}{l}
-B u=\lambda u, \quad x \in(0, L), \\
\alpha_{1} u(0)-\beta_{1} u^{\prime}(0)=0, \\
\alpha_{2} u(L)+\beta_{2} u^{\prime}(L)=0,
\end{array}\right.
$$

there exists a principal eigenvalue $\lambda_{1}>0$ such that $\left|\lambda_{1}\right| \geq\left|\lambda_{n}\right|$ for all other eigenvalues $\lambda_{n}$ of $-B$ [13, Theorem 1]. If $\left\{\lambda_{n}\right\}_{n \in \mathbb{N}}$ are the eigenvalues of $-B$, then $\left\{-\lambda_{n}\right\}_{n \in \mathbb{N}}$ are the eigenvalues of $B$. Therefore, $\left|\lambda_{1}\right| \geq\left|\lambda_{n}\right|$ implies that $\left|-\lambda_{1}\right| \geq\left|-\lambda_{n}\right|$, so $-\lambda_{1}<0$ is the principal eigenvalue of $B$ and $s(B)<0$.

Note that $B$ is a resolvent-positive operator because it generates the positive analytic semigroup $U_{B}(t)=e^{B t}$ on $Z$ [30, Theorem 3.12]. In addition, $C$ is a positive operator, so $A=B+C$ is a positive perturbation of $B$. Moreover, the operator $A$ is also resolvent-positive as it generates a positive analytic semigroup $U_{A}(t)=e^{t A}$ on $X$. Therefore, we conclude from Theorem 2.9 [30, Theorem 3.5] that $s(A)$ has the same sign as $r\left(-C B^{-1}\right)-1=r(\Gamma)-1=$ $R_{0}-1$.

Appendix B. Green's function. Green's function for the ordinary differential equation for the next generation operator (A.7) is given by the solution $k(x, y)$ to $(2.14)$ subject to the boundary conditions $\alpha_{1} k(0, y)-\beta_{1} k^{\prime}(0, y)=0, \alpha_{2} k(L, y)+\beta_{2} k^{\prime}(L, y)=0$ for a fixed $y \in(0, L)$. Then $k(x, y)$ is given by

$$
k(x, y)=k_{1}(x, y) u(y-x)+k_{2}(x, y) u(x-y)
$$

Copyright $\odot$ by SIAM. Unauthorized reproduction of this article is prohibited. 
(see (2.9) in Chapter 3 in [27] or (2-11) in Chapter 7-2 in [11]), where $u(z)$ is the Heaviside function

$$
u(z)=\left\{\begin{array}{l}
0, z<0 \\
1, z \geq 0
\end{array}\right.
$$

and

$$
\begin{aligned}
& k_{1}(x, y)=C_{1}(y) e^{r_{1} x}+C_{2}(y) e^{r_{2} x} \\
& k_{2}(x, y)=C_{3}(y) e^{r_{1} x}+C_{4}(y) e^{r_{2} x},
\end{aligned}
$$

where $r_{1,2}=a \pm \sqrt{a^{2}+4 D v} /(2 D), C_{1}(y), C_{2}(y), C_{3}(y)$, and $C_{4}(y)$ are constants depending on $y$. To specify the $C_{i}$ 's, we use the boundary conditions

$$
\begin{aligned}
& \alpha_{1} k_{1}(0, y)-\beta_{1} k_{1}^{\prime}(0, y)=0 \\
& \alpha_{2} k_{2}(L, y)+\beta_{2} k_{2}^{\prime}(L, y)=0
\end{aligned}
$$

and the following jump discontinuity conditions:

$$
\begin{aligned}
k_{1}(y, y) & =k_{2}(y, y), \\
-D\left(k_{2}^{\prime}(y, y)-k_{1}^{\prime}(y, y)\right) & =1 .
\end{aligned}
$$

Green's function under hostile boundary conditions. For hostile boundary conditions, we find that

$$
\begin{aligned}
C_{1}(y) & =\frac{\exp \left[-\left(r_{1}+r_{2}\right) y\right]\left(\exp \left[L r_{2}+r_{1} y\right]-\exp \left[L r_{1}+r_{2} y\right]\right)\left(-a+D r_{2}\right)}{D\left(r_{1}-r_{2}\right)\left(a \exp \left[L r_{1}\right]-a \exp \left[L r_{2}\right]+D \exp \left[L r_{2}\right] r_{1}-D \exp \left[L r_{1}\right] r_{2}\right)}, \\
C_{2}(y) & =\frac{\exp \left[-\left(r_{1}+r_{2}\right) y\right]\left(\exp \left[L r_{2}+r_{1} y\right]-\exp \left[L r_{1}+r_{2} y\right]\right)\left(a-D r_{1}\right)}{D\left(r_{1}-r_{2}\right)\left(a \exp \left[L r_{1}\right]-a \exp \left[L r_{2}\right]+D \exp \left[L r_{2}\right] r_{1}-D \exp \left[L r_{1}\right] r_{2}\right)}, \\
C_{3}(y) & =\frac{\exp \left[L r_{2}-(r 1+r 2) y\right]\left(a \exp \left[r_{1} y\right]-a \exp \left[r_{2} y\right]+D \exp \left[r_{2} y\right] r_{1}-D \exp \left[r_{1} y\right] r_{2}\right)}{D\left(-r_{1}+r_{2}\right)\left(a \exp \left[L r_{1}\right]-a \exp \left[L r_{2}\right]+D \exp \left[L r_{2}\right] r_{1}-D \exp \left[L r_{1}\right] r_{2}\right)}, \\
C_{4}(y) & =\frac{\exp \left[L r_{1}-\left(r_{1}+r_{2}\right) y\right]\left(-a \exp \left[r_{1} y\right]+a \exp \left[r_{2} y\right]-D \exp \left[r_{2} y\right] r_{1}+D \exp \left[r_{1} y\right] r_{2}\right)}{D\left(r_{1}-r_{2}\right)\left(-a \exp \left[L r_{1}\right]+a \exp \left[L r_{2}\right]-D \exp \left[L r_{2}\right] r_{1}+D \exp \left[L r_{1}\right] r_{2}\right)}
\end{aligned}
$$

Green's function under Danckwert's boundary conditions. For Danckwert's boundary conditions, we find that

$$
\begin{aligned}
C_{1}(y) & =\frac{\exp \left[-\left(r_{1}+r_{2}\right) y\right]\left(a-D r_{2}\right)\left(-\exp \left[L r_{1}+r_{2} y\right] r_{1}+\exp \left[L r_{2}+r_{1} y\right] r_{2}\right)}{D\left(r_{1}-r_{2}\right)\left(D\left(\exp \left[L r_{1}\right]-\exp \left[L r_{2}\right]\right) r_{1} r_{2}+a\left(-\exp \left[L r_{1}\right] r_{1}+\exp \left[L r_{2}\right] r_{2}\right)\right)}, \\
C_{2}(y) & =\frac{\exp \left[-\left(r_{1}+r_{2}\right) y\right]\left(a-D r_{1}\right)\left(\exp \left[L r_{1}+r_{2} y\right] r_{1}-\exp \left[L r_{2}+r_{1} y\right] r_{2}\right)}{D\left(r_{1}-r_{2}\right)\left(D\left(\exp \left[L r_{1}\right]-\exp \left[L r_{2}\right]\right) r_{1} r_{2}+a\left(-\exp \left[L r_{1}\right] r_{1}+\exp \left[L r_{2}\right] r_{2}\right)\right)}, \\
C_{3}(y) & =\frac{-\exp \left[L r_{2}-\left(r_{1}+r_{2}\right) y\right] r_{2}\left(-a \exp \left[r_{1} y\right]+a \exp \left[r_{2} y\right]-D \exp \left[r_{2} y\right] r_{1}+D \exp \left[r_{1} y\right] r_{2}\right)}{D\left(r_{1}-r_{2}\right)\left(D\left(\exp \left[L r_{1}\right]-\exp \left[L r_{2}\right]\right) r_{1} r_{2}+a\left(-\exp \left[L r_{1}\right] r_{1}+\exp \left[L r_{2}\right] r_{2}\right)\right)} \\
C_{4}(y) & =\frac{-\exp \left[L r_{1}-\left(r_{1}+r_{2}\right) y\right] r_{1}\left(a \exp \left[r_{1} y\right]-a \exp \left[r_{2} y\right]+D \exp \left[r_{2} y\right] r_{1}-D \exp \left[r_{1} y\right] r_{2}\right)}{D\left(r_{1}-r_{2}\right)\left(D\left(\exp \left[L r_{1}\right]-\exp \left[L r_{2}\right]\right) r_{1} r_{2}+a\left(-\exp \left[L r_{1}\right] r_{1}+\exp \left[L r_{2}\right] r_{2}\right)\right)}
\end{aligned}
$$

Copyright (C) by SIAM. Unauthorized reproduction of this article is prohibited. 
Acknowledgments. The authors wish to thank Frank Hilker, Ed McCauley, and Andrew Paul for fruitful discussions. HWM is an Alberta Ingenuity Scholar. YJ gratefully acknowledges support from the Alberta Sustainable Resource Development and the Alberta Water Research Institute. JJ gratefully acknowledges sabbatical support from the University of Alberta and Harvey Mudd College. He also thanks the Centre for Mathematical Biology and the Department of Mathematics and Statistics at the University of Alberta for their support and generous hospitality. MAL gratefully acknowledges a Canada Research Chair and NSERC Discovery grant. We thank two anonymous referees for helpful comments that greatly improved the paper.

\section{REFERENCES}

[1] D. J. Allan And M. M. Castillo, Stream Ecology, Structure and Function of Running Waters, 2nd ed., Springer, Berlin, 2007.

[2] K. E. Anderson, A. J. Paul, E. McCauley, L. J. Jackson, J. R. Post, and R. M. Nisbet, Instream flow needs in streams and rivers: The importance of understanding ecological dynamics, Frontiers in Ecology and the Environment, 4 (2006), pp. 309-318.

[3] J. E. Byers And J. M. Pringle, Going against the flow: Retention, range limits and invasions in advective environments, Marine Ecology Progress Series, 313 (2006), pp. 27-41.

[4] R. S. Cantrell and C. Cosner, Spatial Ecology via Reaction-Diffusion Equations, Wiley, New York, 2003.

[5] H. Caswell, Matrix Population Models: Construction, Analysis, and Interpretation, 2nd ed., Sinauer Associates, Sunderland, MA, 2002.

[6] F. Chatelin, The spectral approximation of linear operators with applications to the computation of eigenelements of differential and integral operators, SIAM Rev., 23 (1981), pp. 495-522.

[7] J. M. Cushing And Y. Zhou, The net reproductive value and stability in matrix population models, Natur. Resource Modeling, 8 (1994), pp. 297-333.

[8] T. DE CAMINO-BECK AND M. A. LEWIS, A new method for calculating net reproductive rate from graph reduction with applications to the control of invasive species, Bull. Math. Biol., 69 (2007), pp. 13411354.

[9] O. Diekmann, J. A. P. Heesterbeek, and J. A. J. Metz, On the definition and the computation of the basic reproductive ratio $r_{0}$ in models for infectious diseases in heterogeneous populations, J. Math. Biol., 28 (1990), pp. 365-382.

[10] Y. Du, Order structure and topological methods in nonlinear partial differential equations, vol. 1, in Maximum Principles and Applications of Partial Differential Equations and Applications, World Scientific, River Edge, NJ, 2006.

[11] R. Guenther And J. Lee, Partial Differential Equations of Mathematical Physics and Integral Equations, Dover, New York, 1996.

[12] J. A. P. Heesterbeek, A brief history of $r_{0}$ and a recipe for its calculation, Acta Biotheoretica, 50 (2002), pp. 189-204.

[13] P. Hess And T. Kato, On some linear and nonlinear eigenvalue problems with an indefinite weight function, Comm. Partial Differential Equations, 5 (1980), pp. 999-1030.

[14] F. Hirsch And G. Lacombe, Elements of Functional Analysis, Graduate Texts in Math. 192, Springer, Berlin, 1999.

[15] S. Humphries And G. D. Ruxton, Is there really a drift paradox?, J. Animal Ecology, 71 (2002), pp. 151-154.

[16] M. KRKošEK AND M. A. Lewis, An $R_{0}$ theory for source-sink dynamics with application to Dreissena, Theoretical Ecology, 3 (2010), pp. 25-43.

[17] M. A. Lewis, T. Hillen, And F. Lutcher, Spatial dynamics in ecology, vol. 14, in Mathematical Biology, Institute for Advanced Study/Park City Mathematics Institute, Princeton, NJ, 2009.

Copyright (c) by SIAM. Unauthorized reproduction of this article is prohibited. 
[18] Y. LOU AND X.-Q. ZhaO, A reaction-diffusion malaria model with incubation period in the vector population, J. Math. Biol., 62 (2011), pp. 543-568.

[19] F. Lutscher, M. A. Lewis, And E. MCCauley, Effects of heterogeneity on spread and persistence in rivers, Bull. Math. Biol., 68 (2006), pp. 2129-2160.

[20] F. Lutscher, E. MCCAuley, And M. A. Lewis, Spatial patterns and coexistence mechanisms in systems with unidirectional flow, Theoretical Population Biology, 71 (2007), pp. 267-277.

[21] K. MüLleR, Investigations of the Organic Drift in North Swedish Streams, Technical report, Institute for Freshwater Research, Drottningholm, Sweden, 1954.

[22] E. Pachepsky, F. Lutscher, R. M. Nisbet, And M. A. Lewis, Persistence, spread and the drift paradox, Theoretical Population Biology, 67 (2005), pp. 61-73.

[23] B. D. Richter, D. Braun, M. Mendelson, and L. Master, Threats to imperiled freshwater fauna, Conservation Biology, 11 (1997), pp. 1081-1093.

[24] A. L. Shanks And G. L. Eckert, Population persistence of California current fishes and benthic crustaceans: A marine drift paradox, Ecological Monographs, 75 (2005), pp. 505-524.

[25] H. L. Smith, Monotone dynamical systems: An introduction to the theory of competitive and coopertative systems, in Math. Surveys Monogr. 41, American Mathematical Society, Providence, RI, 1995.

[26] D. Speirs And W. Gurney, Population persistence in rivers and estuaries, Ecology, 82 (2001), pp. 12191237.

[27] I. Stakgold, Green's Functions and Boundary Value Problems, 2nd ed., Wiley, New York, 1998.

[28] P. Steffler And J. Blackburn, Two-Dimensional Depth Averaged Models of River Hydrodynamics and Fish Habitat: Introduction to Depth Averaged Models and User's Manual, Technical report, University of Alberta, AB, Canada, 2002.

[29] R. Tharme, A global perspective on environmental flow assessment: Emerging trends in the development and application of environmental flow methodologies for rivers, River Res. Appl., 19 (2003), pp. 397441.

[30] H. THIEME, Spectral bound and reproductive number for infinite-dimensional population structure and time heterogeneity, SIAM J. Appl. Math., 70 (2009), pp. 188-211.

[31] N. K. Vaidya, F. Wang, And X. Zou, Avian influenza dynamics in wild birds with bird mobility and spatial heterogeneous environment, Dyn. Contin., Discrete Impuls. Syst., accepted (2011).

[32] P. VAn Den Driessche And J. Watmough, Reproduction numbers and sub-threshold endemic equilibria for compartmental models of disease transmission, Math. Biosci., 180 (2002), pp. 29-48.

[33] W. Wang And X.-Q. ZhaO, A nonlocal and time-delayed reaction-diffusion equation model of dengue transmission, SIAM J. Appl. Math., 71 (2011), pp. 147-168.

Copyright ( $\odot$ by SIAM. Unauthorized reproduction of this article is prohibited. 